\title{
Twisting Segal's K-Homology Theory
}

\author{
Dai Tamaki \\ Department of Mathematical Sciences, Shinshu University, Matsumoto, 390-8621, \\ Japan \\ rivulus@math.shinshu-u.ac.jp
}

\section{Introduction}

The aim of this article is twofold:

1. we give a quick introduction to twisted $K$-theory and, more generally, twisted homology and cohomology theories from a homotopy theoretic point of view, and

2. we construct a twisted version of Segal's connective $K$-homology theory.

The first half of this article is based on talks delivered by the author during the conference "Noncommutative Geometry and Physics 2008 - $K$-theory and D-brane -". The basic idea of twisting generalized cohomology theories already appeared in the paper AS04] by Atiyah and Segal, in which a modern treatment of twisted $K$-theory was introduced. Their construction is based on a homotopy theoretic point of view, i.e. as cohomology theories twisted by automorphisms of representing spectra. Nowadays algebraic topologists regard twisted (co)homology theories as (co)homology theories defined by bundles of spectra. See, for example, a paper by C.L. Douglas [Dou06]. A more systematic study was done by Waldmüller in Wal. The first half of this article is intended to be an exposition of basic ideas behind these abstract approaches to twisted (co)homology theories for those who are not familiar with homotopy theory.

Descriptions of $K$-theory depend on the context. The periodic cohomological $K$-theory of a compact Hausdorff space $X$ can be described in terms of

- vector bundles over $X$,

- homotopy classes of maps from $X$ to the space of Fredholm operators on a separable infinite dimensional Hilbert space, and

- the $C^{*}$-algebra of continuous functions on $X$.

Corresponding twisted versions have been studied intensively. 
Recent interests in twisted $K$-theory are based on the observation of Witten Wit98] that $D$-brane charges give rise to elements of twisted $K$ theory. For this purpose, however, recent results of Reis, Szabo, and Valentino RS06, RSV] suggest to use homological $K$-theory instead of cohomological $K$-theory.

Although we can always construct a homology theory corresponding to a given cohomology theory by using homotopy theoretic methods, there aren't many concrete descriptions of $K$-homology groups. The periodic $K$-homology theory can be described by using $C^{*}$-algebras or geometric cycles of Baum and Douglas BD82. A twisted version of Baum-Douglas $K$-homology theory was constructed by B.-L. Wang in Wan.

In an intriguing paper [Seg77, G.B. Segal found a description of the connective version of homological $K$-theory by categorifying the Dold-Thom description of the ordinary integral homology DT58. As a concrete example of a twisted homology theory, we construct a twisted version of Segal's connective $K$-homology theory in the second half of this article. The construction is based on a new description of Segal's connective $K$-homology theory in terms of inifinite dimensional projective space bundles.

This paper is organized as follows:

- 92 is devoted to an exposition of twisted homology and cohomology theories from a homotopy theoretic point of view.

After describing generalized cohomology and homology theories in terms of spectra in $\$ 2.1$ and $\$ 2.2$ we explain ideas lying behind twisted cohomology theories in $\$ 2.3$ and give a modern homotopy theoretic way of studying twisted cohomology theories in $\$ 2.4$. The Atiyah-Segal twisting of the complex $K$-theory is briefly recalled in 2.5 .

- In \$3.1, we introduce a new description of the complex version of Segal's $K$-homology theory and then our twisted version of $K$-homology functor. We prove our construction gives rise to a twisted homology theory in the sense of $\$ 2.4$ in $\$ 3.2$. Our proof is base on a homotopy theoretic result proved in a separate paper [Tam].

Acknowledgement. Ideas in the second half of this paper were developed when the author was preparing his talks for various seminars. The idea of using infinite dimensional projective space to describe Segal's functor $k u(X ; H)$ was found when the author was preparing for talks in a seminar at Tateshina in 2004. The formulation of Theorem 10 was obtained when the author was preparing for a talk in a seminar at Kinosaki in 2005. And the author realized that his model of Segal's $K$-homology possesses a natural way of twisting during the conference on $D$-branes and $K$-theory held at Shonan in 2008. The author is grateful to organizers of all these meetings, especially M. Furuta, Moriyoshi, D. Kishimoto, S. Kaji, T. Matsuoka, A. Kono, and T. Kato, for inviting him to give talks.

This work is partially supported by Grants-in-Aid for Scientific Research, Ministry of Education, Culture, Sports, Science and Technology, Japan: 17540070. 


\section{A Quick Introduction to Twisted Homology and Cohomology Theories}

The aim of this section is to introduce the twisted $K$-theory and, more generally, twisted homology and cohomology theories from a homotopy theoretic point of view.

\subsection{From Geometry to Homotopy Theory}

Let us begin with a brief history of untwisted homology and cohomology theories. The idea of homology is due to H. Poincaré Poi96 who initiated the study of smooth manifolds in terms of submanifolds and boundary relations among them. The properties of homology groups have been axiomatized by Eilenberg and Steenrod ES52] as a functor

\section{$H_{*}$ : Pairs of Spaces $\longrightarrow$ Graded Abelian Groups}

satisfying certain conditions.

R. Thom Tho54 found an alternative way to realize Poincaré's idea in his study of the realization problem of homology classes by submanifolds. Thom's idea was extended by Atiyah [Ati61] as a functor

$$
M O_{*}: \text { Spaces } \longrightarrow \text { Graded Abelian Groups }
$$

having properties similar to the Eilenberg-Steenrod axioms.

Atiyah, together with Hirzebruch [AH59, found another functor

$$
K^{*}: \text { Spaces }^{\text {op }} \longrightarrow \text { Graded Abelian Groups }
$$

by importing an idea of Grothendieck in algebraic geometry to topology, where $(-)^{\text {op }}$ denotes the opposite category. Although their functor is contravariant, it has properties analogous to the Eilenberg-Steenrod axioms.

In order to understand these functors in a unified way, E. Brown Bro62 introduced a set of axioms for cohomology theories by modifying the cohomological version of the axioms of Eilenberg and Steenrod. Precisely speaking, there are two ways to axiomatize generalized cohomology theories: cohomology theories for pairs and reduced cohomology theories for based spaces. Let us consider reduced versions here. Thus a generalized cohomology theory is a functor

$$
\tilde{E}^{*}: \operatorname{Spaces}_{*}^{\mathrm{op}} \longrightarrow \text { Graded Abelian Groups }
$$

equipped with natural isomorphisms

$$
\Sigma: \tilde{E}^{n}(X) \longrightarrow \tilde{E}^{n+1}(\Sigma X)
$$

satisfying certain conditions, where Spaces $_{*}$ is the category of based spaces and basepoint preserving continuous maps and $\Sigma X$ is the reduced suspension of $X$.

Brown also proved Bro65] that any such a generalized cohomology theory can be represented by a sequence of based spaces. 
Theorem 1 (E.H. Brown, Jr.). For any reduced cohomology theory $\widetilde{E}^{*}(-)$, there exists a sequence of based spaces

$$
\cdots, E_{n}, E_{n+1}, \cdots
$$

equipped with based maps $\varepsilon_{n}: \Sigma E_{n} \rightarrow E_{n+1}$ such that, for any $C W$ complex $X$, we have natural isomorphisms

$$
\widetilde{E}^{n}(X) \cong\left[X, E_{n}\right]_{*}
$$

for all $n$ and that the suspension isomorphism (1) is induced by the composition

$$
\left[X, E_{n}\right]_{*} \longrightarrow\left[\Sigma X, \Sigma E_{n}\right]_{*} \longrightarrow\left[\Sigma X, E_{n+1}\right]_{*},
$$

where $[-,-]_{*}$ denotes the set of based homotopy classes of based maps.

Such a sequence $E=\left\{E_{n}\right\}_{n \in \mathbb{Z}}$ is called a spectrum. Conversely any spectrum $E$ gives rise to a reduced cohomology theory.

Proposition 1. For any spectrum E, define

$$
\widetilde{E}^{n}(X)=\operatorname{colim}_{k}\left[X, \Omega^{k}\left(E_{n+k} \wedge X\right)\right]_{*}
$$

then $\widetilde{E}^{*}(-)$ is a reduced cohomology theory.

Brown's representability theorem and the above Proposition say the study of generalized cohomology theories can be reduced to the homotopy theory of spectra.

What do we mean by the homotopy theory of spectra? Spectra form a category Spectra. After the introduction of the notion of model category by Quillen Qui67, a homotopy theory in a category C means a model structure on $\mathbf{C}$. A model structure on a category $\mathbf{C}$ consists of three classes of morphisms; fibrations, cofibrations, and weak equivalences, and two ways to factor any morphism into a composition of two morphisms. A precise definition of model category can be found in [DS95, Hov99, Hir03].

It took more than 30 years after Brown proved his representablity theorem for topologists to find a good model category of spectra. One of them was constructed by Elmendorf, Kriz, Mandell, and May in EKMM97. Symmetric spectra and orthogonal spectra introduced by Hovey, Shipley, and Smith HSS00 and by Mandell and May [MM02, respectively, also give us useful model categories of spectra. In fact, symmetric spectra are used in a construction of spectrum representing $K K$-theory of $C^{*}$-categories by Mitchener Mit and orthogonal spectra are used by Bunke, Joachim, and Stolz BJS03. in their construction of a spectrum representing $K K$-theory.

We do not intend to go into details of model categories nor spectra here. But the existence of a good model category of spectra guarantees that we can treat spectra as though they are spaces and we have notions analogous to 
homotopy equivalences. We should also note here that the category of spectra is symmetric monoidal, i.e. there is a way to produce a "smash product" $E \wedge F$ of two spectra $E$ and $F$. This operation $\wedge$ is analogous to the smash product

$$
X \wedge Y=X \times Y / X \times\{*\} \cup\{*\} \times Y
$$

of two based spaces $X$ and $Y$. In particular, we can smash a spectrum $E$ and a space $X$ to obtain a spectrum $E \wedge X$.

\subsection{Linear Functors and Homology Theories}

Compared to cohomology theories, it is not easy to find a good description of homology theories. When a cohomology theory $E^{*}(-)$ is represented by a spectrum $E$, G.W. Whitehead Whi62 found a way to construct a corresponding (reduced) connective homology theory $\widetilde{E}_{*}(-)$ by

$$
\widetilde{E}_{n}(X)=\operatorname{colim}_{k} \pi_{n+k}\left(E_{k} \wedge X\right) \cong \pi_{n}\left(\Omega^{\infty}(E \wedge X)\right),
$$

where

$$
\Omega^{\infty}: \text { Spectra } \longrightarrow \text { Spaces }_{*}
$$

is a functor which produces an infinite loop space from a spectrum by

$$
\Omega^{\infty} E=\operatorname{colim}_{k} \Omega^{k} E_{k} .
$$

Thus algebraic properties of $\widetilde{E}_{*}(-)$ come from homotopy-theoretic properties of the functor

$$
\Omega^{\infty}(E \wedge(-)): \text { Spaces }_{*} \longrightarrow \text { Spaces }_{*} .
$$

One of the most important properties of such functors is linearity.

Definition 1. A functor

$$
F: C \longrightarrow D
$$

between model categories is called a homotopy functor if it maps weak equivalences to weak equivalences.

Definition 2. Let $\boldsymbol{C}$ be a model category with a 0-object $*$. A homotopy functor

$$
F: C \longrightarrow \text { Spaces }_{*}
$$

is called linear if it satisfies the following conditions:

1. $F(*) \underset{w}{\simeq}$, where $\underset{w}{\simeq}$ means there is a weak equivalence between them.

2. For a family of objects $\left\{X_{\alpha}\right\}_{\alpha \in A}$ with $* \hookrightarrow X_{\alpha}$ a cofibration, we have a weak equivalence

$$
\prod_{\alpha \in A} F\left(X_{\alpha}\right) \underset{w}{\simeq} F\left(\bigvee_{\alpha \in A} X_{\alpha}\right) \text {. }
$$


3. For a cofibration $A \rightarrow X$ with cofiber $X / A$, we have a quasifibration

$$
F(X) \longrightarrow F(X / A)
$$

with fiber $F(A)$.

Recall that a quasifibration is a map which induces a long exact sequence of homotopy groups similar to that of a fibration.

Goodwillie developed a technique so-called "calculus of homotopy functors" and studied general properties of homotopy functors extensively. For example, Goodwillie proved in Goo03 that, for any linear homotopy functor

$$
F: \operatorname{Spaces}_{*} \longrightarrow \text { Spaces }_{*},
$$

there exists a spectrum $E_{F}$ with

$$
F(X) \underset{w}{\simeq} \Omega^{\infty}\left(E_{F} \wedge X\right) .
$$

Thus connective homology theories are essentially equivalent to linear functors.

One of the first examples of such a functor was discovered by Dold and Thom DT58.

Example 1. For a based space $X$, define

$$
\operatorname{SP}^{\infty}(X)=\left(\coprod_{n} X^{n} / \Sigma_{n}\right) / \sim
$$

where $\Sigma_{n}$ is the symmetric group of $n$ letters and the relation $\sim$ is defined by

$$
\left[x_{1}, \cdots, x_{n}, *\right] \sim\left[x_{1}, \cdots, x_{n}\right] .
$$

$\mathrm{SP}^{\infty}(X)$ is called the infinite symmetric product of $X$. Then a famous theorem of Dold and Thom [DT58] says

$$
\mathrm{SP}^{\infty}: \operatorname{Spaces}_{*} \longrightarrow \text { Spaces}_{*}
$$

is a linear functor.

They also proved that this functor corresponds to the ordinary integral homology theory

$$
\pi_{n}\left(\operatorname{SP}^{\infty}(X)\right) \cong \widetilde{H}_{n}(X ; \mathbb{Z})
$$

The right hand side could be described as $\pi_{n}\left(\Omega^{\infty}(H \mathbb{Z} \wedge X)\right)$ by using the integral Eilenberg-Mac Lane spectrum $H \mathbb{Z}$, but the description by the infinite symmetric product is much nicer and more understandable. 
Example 2. Let $H$ be a real inner product space of countable dimension. Segal Seg77 introduced a functor

$$
k o(-; H): \mathbf{S p a c e s}_{*} \longrightarrow \text { Spaces }_{*}
$$

defined by

$$
k o(X ; H)=\left\{\begin{array}{l|l}
{\left[V_{x_{1}}, V_{x_{2}}, \cdots, V_{x_{j}}\right]} & \begin{array}{l}
x_{i} \in X, \\
V_{x_{i}} \subset H: \text { finite dim. } \\
x_{i} \neq x_{i^{\prime}} \text { if } i \neq i^{\prime}, \\
V_{x_{i}} \perp V_{x_{i^{\prime}}} \text { if } i \neq i^{\prime}
\end{array}
\end{array}\right\}
$$

with suitable topology.

He proved that $k o(-; H)$ is linear and gives rise to the connective $K O$ homology theory.

Note that $\mathrm{SP}^{\infty}(X)$ can be regarded as a free topological monoid generated by $X$ and an element of $\mathrm{SP}^{\infty}(X)$ is a formal sum of points in $X$. By grouping the same points together, such an element can be written as a collection of positive integers labelled by points in $X$

$$
\left[n_{x_{1}}, n_{x_{2}}, \cdots, n_{x_{j}}\right]
$$

where $x_{i} \in X, n_{x_{i}} \in \mathbb{N}$, and $x_{i} \neq x_{k}$ if $i \neq k$.

As we can see from this description, Segal's functor gives us a straightforward way to extend the dimension function

$$
\operatorname{dim}: \text { Vector Spaces } \longrightarrow \mathbb{N} \cup\{0\}
$$

to a natural transformation of linear functors.

A natural question is how to define a twisting of the complex version of Segal's $K$-homology theory. When a homology theory is desribed in terms of a spectrum, there is a natural way to twist. For a linear functor with a concrete description, however, there should be a concrete way to twist it. We propose an answer to this question in 93 .

\subsection{What is a Twisted Cohomolgoy Theory?}

Let us now consider twistings of a given cohomology theory. We will consider homology theories later.

One of the origins of the twisted $K$-theory is a paper by Donovan and Karoubi DK70] entitled "Graded Brauer groups and $K$-theory with local coefficients". Ordinary cohomology groups with local coefficients are usually defined in terms of cochains. There are no cochians for $K$-theory. In order to find a definition of $K$-theory with local coefficients, we need space or spectrum level constructions. 
How can we describe the ordinary cohomology groups with local coeffcients without cochains? Given a path-connected space $X$, a local coefficient system $\underline{M}$ of Abelian groups on $X$ is nothing but a structure of $\pi_{1}(X)$-module on an Abelian group $M$. Or a group homomorphism

$$
\varphi: \pi_{1}(X) \longrightarrow \operatorname{Aut}(M) .
$$

By applying the classifying space functor $B(-)$, we obtain a map

$$
B \varphi: B \pi_{1}(X) \longrightarrow B \operatorname{Aut}(M)
$$

and we have a corresponding principal Aut $(M)$-bundle

$$
E_{\varphi}=B \varphi^{*}(E \operatorname{Aut}(M)) \longrightarrow B \pi_{1}(X) .
$$

Among several well-known constructions of the classfying spaces of groups, one of the most convenient is Milgram's construction [Mil67].

Theorem 2. There are functors

$$
\begin{aligned}
& E: \text { Topological Monoids } \longrightarrow \text { Spaces }_{*} \\
& B: \text { Topological Monoids } \longrightarrow \text { Spaces }_{*}
\end{aligned}
$$

and a natural transformation

$$
p: E \longrightarrow B
$$

having the following properties:

1. The homotopy groups of $E(M)$ is trivial for any $M$.

2. For a topological group $G$ with $(G, e)$ a strong NDR pair,

$$
p_{G}: E(G) \longrightarrow B(G)
$$

is a principal $G$-bundle.

3. The projections induce a natural homeomorphism

$$
B(M \times N) \cong B M \times B N .
$$

4. For a topological Abelian monoid $M$, the monoid structure on $M$ induces a monoid structure on BM. When $M$ is a topological Abelian group, so is $B M$.

The action of $\operatorname{Aut}(M)$ on $M$ induces an action on $B M$, hence on the topological Abelian group $B^{n} M$ for $n \in \mathbb{N}$. Thus, the classifying map

$$
\pi_{X}: X \longrightarrow B \pi_{1}(M)
$$

of the universal covering over $X$ induces a bundle

$$
p_{X}^{B^{n} M}: \pi_{X}^{*}\left(E \pi_{1}(M)\right) \times \operatorname{Aut}(M) B^{n} M \longrightarrow X
$$

over $X$ with fiber $B^{n} M$.

It is easy to see that we obtain the cohomology of $X$ with local coefficients as the group of homotopy classes of sections of this bundle. 
Definition 3. For a continuous map $p: E \rightarrow X$, the space of sections of $p$ is denoted by $\Gamma(p)$.

Proposition 2. We have a natural isomorphism

$$
H^{n}(X ; \underline{M}) \cong \pi_{0}\left(\Gamma\left(p_{X}^{B^{n} M}\right)\right)
$$

for any $C W$ complex $X$, where $\underline{M}$ is the local coefficient system over $X$ associated with a given action of $\pi_{1}(X)$ on $M$.

Proof. The skeletal filtration on $X$ induces a spectral sequence converging to $\bigoplus_{n} \pi_{*}\left(\Gamma\left(p_{X}^{B^{n} M}\right)\right)$. Since $B^{n} M$ is an Eilenberg-Mac Lane space, the $E^{1}$-term is the cellular cochain complex with coefficients in $\underline{M}$. The spectral sequence collapses at the $E^{2}$-term and we obtain the desired isomorphism.

This proposition says that a twisting of $H^{n}(X ; M)=\left[X, B^{n} M\right]$ is given by an action of a group $G=\operatorname{Aut}(M)$ on $B^{n} M$ and a map

$$
\pi_{X}: X \longrightarrow B G \text {. }
$$

Definition 4. Let $F:$ Spaces $^{\mathrm{op}} \rightarrow$ Abelian Groups be a functor represented by a space BF. A twisting of $F(X)$ is given by an action of a topological group $G$ on $B F$ and a map

$$
\varphi: X \longrightarrow B G .
$$

The functor twisted by $G$ and $\varphi$ is defined by

$$
{ }_{G, \varphi} F(X)=\pi_{0}\left(\Gamma\left(\varphi^{*}(E G) \times_{G} B F\right)\right) .
$$

Note that

$$
F(X)=\pi_{0}(\operatorname{Map}(X, B F))=\pi_{0}(\Gamma(X \times B F \rightarrow X)) .
$$

A twisting is a twisting of the trivial bundle $X \times B F$.

Suppose we have a (reduced) cohomology theory

$$
\widetilde{E}^{*}(-): \text { Spaces }_{*}^{\mathrm{op}} \longrightarrow \text { Graded Abelian Groups }
$$

represented by a spectrum $E$. Although each $\widetilde{E}^{n}(X)$ is representable,

$$
\widetilde{E}^{n}(X) \cong\left[X, E_{n}\right]_{*},
$$

it is representable as a functor on the category of based spaces. In order to define a twisting of a cohomology theory, we first need to understand twistings of functors on the category of based spaces, which is the subject of the next subsection. 


\subsection{Generalized Twisted Homology and Cohomology Theories}

According to Definition 4, a twisting of a representable functor $F(X)=$ $[X, B F]$ is given by an action of a group $G$ on $B F$ and a map

$$
\varphi: X \longrightarrow B F \text {. }
$$

We denoted the twisted functor by $G, \varphi F(X)$, but this is misleading. This is not a functor of $X$. Note that knowing of a map $\varphi$ implies knowing of $X$. We should regard ${ }_{G, \varphi} F(X)$ as a functor of $\varphi$.

Definition 5. Fix a space B. The category of spaces over $B$ is denoted by Spaces $\downarrow B$. Objects are maps $\varphi: X \rightarrow B$ and a morphism $f$ from $\varphi$ to $\psi$ is a commutative diagram

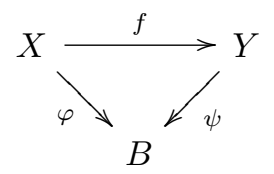

We can rewrite the definition of a twisted functor as follows.

Definition 6. Let $F:$ Spaces $^{\mathrm{op}} \rightarrow$ Abelian Groups be a functor repre- $^{-}$ sented by a space BF. A twisting of $F$ is given by an action of a topological group $G$ on $B F$, i.e. a continuous homomorphism

$$
\tau: G \longrightarrow \operatorname{Homeo}(B F) .
$$

The twisted functor associated with $\tau$ is a functor

$$
{ }_{\tau} F:(\text { Spaces } \downarrow B G)^{\mathrm{op}} \longrightarrow \text { AbelianGroups }
$$

defined by

$$
{ }_{\tau} F(\varphi)=\pi_{0}\left(\Gamma\left(\varphi^{*}(E G) \times{ }_{G} B F\right)\right)
$$

for an object $\varphi: X \rightarrow B G$ in Spaces $\downarrow B G$. When $\tau$ is obvious from the context, we denote it by ${ }_{G} F(\varphi)$.

We can rewrite ${ }_{\tau} F$ as a representable functor.

Lemma 1. We have a natural isomorphism

$$
{ }_{\tau} F(\varphi) \cong \pi_{0}\left(\operatorname{Mor}_{\mathbf{S p a c e s} \downarrow B G}\left(\varphi, p_{G, B F}\right)\right),
$$

where

$$
p_{G, B F}=p_{G} \times_{G} *: E G \times_{G} B F \longrightarrow B G
$$

and $\operatorname{Mor}_{\mathbf{S p a c e s} \downarrow B G}(-,-)$ is the space of morphisms in Spaces $\downarrow B G$. 
Paths in Mor $\operatorname{Spaces}_{\downarrow} B G(-,-)$ define a notion of homotopy in the category Spaces $\downarrow B G$. The above gives us a description of the twisted functor ${ }_{\tau} F$ as a homotopy set in the category Spaces $\downarrow B G$.

The based versioin is analogous. Suppose we have a functor

$$
F: \text { Spaces }_{*}^{\text {op }} \longrightarrow \text { Abelian Groups }
$$

represented by $B F$

$$
F(X) \cong[X, B F]_{*}=\operatorname{Map}_{*}(X, B F) / \widetilde{\sim},
$$

where $\operatorname{Map}_{*}(-,-)$ is the space of base point preserving maps and $\underset{*}{\simeq}$ is the equivalence relation defined by based homotopy.

Definition 7. Let $p: E \rightarrow B$ be a morphism in $\mathbf{S p a c e s}_{*}$. Define

$$
\Gamma_{*}(p)=\Gamma(p) \cap \operatorname{Map}_{*}(B, E) .
$$

With this notation, we have the following expression

$$
F(X)=\Gamma_{*}(X \times B F \rightarrow X) /_{\underset{*}{\sim}} .
$$

Definition 8. Let $F:$ Spaces $_{*}^{o p} \rightarrow$ Abelian Groups be a functor repre- $^{-}$ sented by a based space BF. A twisting of $F$ is given by a based action $\tau$ of a topological group $G$ on BF. The twisted functor associated with $\tau$ is a functor

$$
{ }_{\tau} F:\left(\text { Spaces }_{*} \downarrow B G\right)^{\mathrm{op}} \longrightarrow \text { Abelian Groups }
$$

defined by

$$
{ }_{\tau} F(\varphi)=\Gamma_{*}\left(\varphi^{*}(E G) \times{ }_{G} B F\right) / \widetilde{\sim} .
$$

This functor can be described as a homotopy set in the category of exspaces over $B G$.

Definition 9. Let $B$ be a space. An ex-space over $B$ is a based object in Spaces $\downarrow B$, i.e. a pair $(\varphi, s)$ of an object $\varphi: E \rightarrow B$ and its section $s: B \rightarrow$ $E$. The category of ex-spaces over $B$ is denoted by Spaces $_{B}$.

Since the action $\tau$ of $G$ on $B F$ is base point preserving, the projection

$$
E G \times{ }_{G} B F \longrightarrow B G
$$

has a canonical section. Let us denote this section by $s_{\tau}$. We obtain an object $\left(p_{G, B F}, s_{\tau}\right)$ in $\operatorname{Spaces}_{B G}$. For each based map $\varphi: X \rightarrow B G$, we also have an object $\left(\varphi \vee 1_{B G}, i_{B G}\right)$ in $\operatorname{Spaces}_{B G}$, where $i_{B G}$ is the canonical inclusion and

$$
\varphi \vee 1_{B G}: X \vee B G \longrightarrow B G
$$


Lemma 2. For any object $\varphi: X \rightarrow B G$, we have a natural isomorphism

$$
\Gamma_{*}\left(\varphi^{*}(E G) \times_{G} B F\right) \cong \operatorname{Mor}_{\operatorname{Spaces}_{B G}}\left(\left(\varphi \vee 1_{B G}, i_{B G}\right),\left(p_{G, B F}, s_{\tau}\right)\right) .
$$

Proof. Under the identification

$\operatorname{Map}_{*}\left(X \vee B G, E G \times_{G} B F\right)=\operatorname{Map}_{*}\left(X, E G \times_{G} B F\right) \times \operatorname{Map}_{*}\left(B G, E G \times_{G} B F\right)$,

an element $f$ of $\operatorname{Mor}_{\operatorname{Spaces}_{B G}}\left(\left(\varphi \vee 1_{B G}, i_{B G}\right),\left(p_{G, B F}, s_{\tau}\right)\right)$ is given by a pair of maps

$$
\begin{aligned}
& f_{1}: X \longrightarrow E G \times_{G} B F \\
& f_{2}: B G \longrightarrow E G \times_{G} B F .
\end{aligned}
$$

The condition that $f$ is a morphism in $\operatorname{Spaces}_{B G}$ implies $f_{2}=s_{\tau}$ and $f_{1}$ makes the following diagram commutative

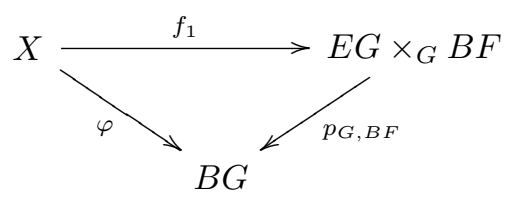

Hence $f_{1}$ defines an element in $\Gamma_{*}\left(\varphi^{*}(E G) \times_{G} B F\right)$.

There is a notion of homotopy in $\mathbf{S p a c e s}_{B G}$. Thus we obtain a description of ${ }_{\tau} F(\varphi)$ as a homotopy set.

Definition 10. For objects $(\varphi, s)$ and $(\psi, t)$ in $\mathbf{S p a c e s}_{B G}$, the set of homotopy classes of morphisms from $(\varphi, s)$ to $(\psi, t)$ is denoted by $[(\varphi, s),(\psi, t)]_{B G}$.

Corollary 1. We have the following natural isomorphism

$$
{ }_{\tau} F(\varphi) \cong\left[\left(\varphi \vee 1_{B G}, i_{B G}\right),\left(p_{G, B F}, s_{\tau}\right)\right]_{B G}
$$

The above argument implies that the functor obtained from a representable functor

$$
F: \text { Spaces }_{*}^{\text {op }} \longrightarrow \text { Abelian Groups }
$$

by a twisting should be regarded as a functor

$$
{ }_{\tau} F: \operatorname{Spaces}_{B G}^{\mathrm{op}} \longrightarrow \text { Abelian Groups. }
$$

Suppose we have a reduced cohomology theory $\widetilde{E}^{*}(-)$ represented by a spectrum $E=\left\{E_{n}\right\}_{n}$. Suppose we have a based action $\tau_{n}$ of a topological group $G$ on $E_{n}$ for each $n$. Then we obtain a sequence of twisted functors

$$
\tau_{n} \widetilde{E}^{n}: \operatorname{Spaces}_{B G}^{\mathrm{op}} \longrightarrow \text { Abelian Groups. }
$$


These functors should satisfy axioms analogous to the axioms for untwisted cohomology theories.

The following is the axioms of cohomology theories on the category Spaces $_{B}$ of ex-spaces over a space $B$. Recall that there are notions of suspension $\Sigma_{B}$, weak equivalences, and cofibrations in Spaces $_{B}$. For basic definitions and properties concerning ex-spaces, see [CJ98] and MS06.

Definition 11. Let $B$ be a space. A (reduced) cohomology theory on Spaces $_{B}$ is a pair of a contravariant functor

$$
\tilde{h}^{*}: \operatorname{Spaces}_{B}^{\text {op }} \longrightarrow \text { Graded Abelian Groups }
$$

and a natural transformation

$$
\Sigma: \tilde{h}^{q} \longrightarrow \tilde{h}^{q+1} \circ \Sigma_{B}
$$

satisfying the following conditions:

1. (Homotopy Invariance) Any weak equivalence

$$
f:(\varphi, s) \longrightarrow(\psi, t)
$$

induces an isomorphism

$$
f^{*}: \tilde{h}^{n}(\psi, t) \stackrel{\cong}{\cong} \tilde{h}^{n}(\varphi, s)
$$

for all $n$.

2. (Exactness) For a cofibration $i:(\varphi, s) \hookrightarrow(\psi, t)$ with cofiber $(\tau, u)$, we have an exact sequence

$$
\tilde{h}^{q}(\tau, u) \stackrel{q^{*}}{\longrightarrow} \tilde{h}^{q}(\psi, t) \stackrel{i^{*}}{\longrightarrow} \tilde{h}^{q}(\varphi, s) .
$$

3. (Suspension) For any $q \in \mathbb{Z}$ and $(\varphi, s)$,

$$
\Sigma: \tilde{h}^{q}(\varphi, s) \longrightarrow \tilde{h}^{q+1}\left(\Sigma_{B}(\varphi, s)\right)
$$

is an isomorphism.

4. (Additivity) For a family of ex-spaces over $B\left\{\left(\varphi_{\alpha}, s_{\alpha}\right)\right\}_{\alpha \in A}$, the inclusions

$$
\left(\varphi_{\alpha}, s_{\alpha}\right) \longrightarrow \bigvee_{\alpha \in A}\left(\varphi_{\alpha}, s_{\alpha}\right)
$$

induce an isomorphism

$$
\tilde{h}^{q}\left(\bigvee_{\alpha \in A}\left(\varphi_{\alpha}, s_{\alpha}\right)\right) \cong \prod_{\alpha \in A} \tilde{h}^{q}\left(\varphi_{\alpha}, s_{\alpha}\right)
$$

if $*_{B} \hookrightarrow\left(\varphi,{ }_{\alpha}, s_{\alpha}\right)$ is a cofibration for all $\alpha$, where $\bigvee_{\alpha \in A}$ is the coproduct in $\operatorname{Spaces}_{B}$. 
Dually we have a corresponding set of axioms for homology theory by reversing arrows and replacing $\prod$ by $\bigoplus$. We omit the definition of homology theory on Spaces $_{B}$, which should be obvious.

As we have seen, given a (reduced) cohomology theory $\widetilde{E}^{*}(-)$ on $\mathbf{S p a c e s}_{*}$ represented by a spectrum $E=\left\{E_{n}\right\}$ and an action of a topological group on $E_{n}$ for each $n$, we obtain a sequence of functors

$$
\tau_{n} \widetilde{E}^{n}: \text { Spaces }_{B G}^{\mathrm{op}} \longrightarrow \text { Abelian Groups. }
$$

In order for this sequence to satisfy the above axioms, we need to impose certain conditions on the actions.

Definition 12. Let $E=\left\{E_{n}\right\}$ be a spectrum and $G$ be a topological group. An action of $G$ on $E$ is a sequence of actions

$$
\tau_{n}: G \longrightarrow \operatorname{Homeo}_{*}\left(E_{n}\right)=\operatorname{Homeo}\left(E_{n}\right) \cap \operatorname{Map}_{*}\left(E_{n}, E_{n}\right)
$$

making the following diagram commutative

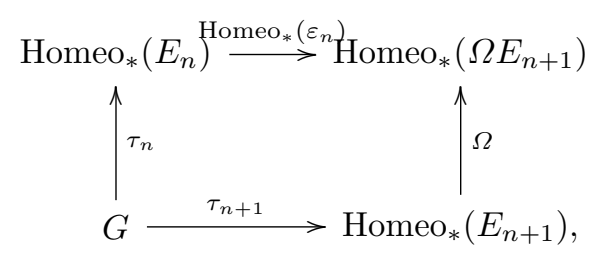

where $\varepsilon_{n}$ are the structure maps of $E$.

Note that the topology of $\operatorname{Homeo}_{*}(Y)$ is defined as the subspace topology under the inclusion

$$
\operatorname{Homeo}_{*}(Y) \stackrel{\Delta}{\longrightarrow} \operatorname{Homeo}_{*}(Y) \times \operatorname{Homeo}_{*}(Y) \stackrel{1_{Y} \times \nu}{\longrightarrow} \operatorname{Map}(Y, Y) \times \operatorname{Map}(Y, Y),
$$

where $\nu: \operatorname{Homeo}_{*}(Y) \rightarrow \operatorname{Homeo}_{*}(Y)$ is the inverse and $\operatorname{Map}(Y, Y)$ is equipped with the compact-open topology.

Given an action of a topological group $G$ on a spectrum $E$, we obtain a sequence of bundles

$$
p_{G, E_{n}}: E G \times_{G} E_{n} \longrightarrow B G .
$$

Since the action of $G$ on $E_{n}$ is base point preserving, we have an object $\left(p_{G, E_{n}}, s_{\tau_{n}}\right)$ in $\operatorname{Spaces}_{B G}$. Under our assumption, the structure map $\varepsilon_{n}$ induces a map

$$
\varepsilon_{n}:\left(p_{G, E_{n}}, s_{\tau_{n}}\right) \longrightarrow \Omega_{B G}\left(p_{G, E_{n+1}}, s_{\tau_{n+1}}\right) .
$$

Thus the sequence $\left\{\left(p_{G, E_{n}}, s_{\tau_{n}}\right)\right\}_{n}$ forms a spectrum in $\mathbf{S p a c e s}_{B G}$. We denote this spectrum by ${ }_{\tau} E$ or ${ }_{G} E$, when the action is obvious from the context.

The following is our definition of twisted cohomology theory. 
Definition 13. Let $\widetilde{E}^{*}(-)$ be a reduced cohomology theory represented by a spectrum E. For an action $\tau$ of a topological group $G$ on $E$, the associated twisted cohomology theory is a functor

$$
{ }_{\tau} \widetilde{E}^{*}: \operatorname{Spaces}_{B G}^{\mathrm{op}} \longrightarrow \text { Graded Abelian Groups }
$$

defined by

$$
{ }_{\tau} \widetilde{E}^{n}(\varphi, s)=\underset{k}{\operatorname{colim}}\left[(\varphi, s), \Omega_{B G}^{k}\left({ }_{\tau} E\right)_{n+k}\right] \mathbf{S p a c e s}_{B G} .
$$

The proof of the following fact is parallel to the standard proof of Proposition 1 .

Proposition 3. Let $E$ be a spectrum and $G$ be a topological group acting on $E$. Then ${ }_{G} \widetilde{E}^{*}(-)$ is a cohomology theory on Spaces $_{B G}$.

Example 3. Let $M$ be an Abelian group. Let $G=\operatorname{Aut}(M)$. Then $G$ acts on the Eilenberg-Mac Lane spectrum $H M$, where

$$
H M_{n}=B^{n} M .
$$

Note that we are using Milgram's construction Mil67] of the classifying space functor so that we can iterate taking $B(-)$.

Then, for $\varphi: X \rightarrow B \operatorname{Aut}(M)$, we obtain a representation of the fundamental group of $X$

$$
\varphi_{*}: \pi_{1}(X) \longrightarrow \pi_{1}(B \operatorname{Aut}(M)) \cong \operatorname{Aut}(M),
$$

i.e. a local coefficient system $\underline{M}$. The twisted cohomology theory associated with the group $\operatorname{Aut}(M)$ is nothing but the cohomology with local coefficient

$$
\operatorname{Aut}(M) \widetilde{H M}^{n}(\varphi) \cong \widetilde{H}^{n}(X ; \underline{M}) .
$$

Now let us consider twistings in a homology theory $\widetilde{E}_{*}(-)$. Suppose $\widetilde{E}_{*}(-)$ is represented by a spectrum $E$

$$
\widetilde{E}_{n}(X)=\operatorname{colim}_{k} \pi_{n}\left(\Omega^{k}\left(E_{k} \wedge X\right)\right) \cong \pi_{n}\left(\Omega^{\infty}(E \wedge X)\right) .
$$

We can extend the functor

$$
\Omega^{\infty}(E \wedge(-)): \text { Spaces }_{*} \longrightarrow \text { Spaces }_{*}
$$

as follows.

Definition 14. Let $\tau$ be an action of a topological group acting $G$ on E. For an object $(\varphi, s)$ in $\mathbf{S p a c e s}_{B G}$, let $s_{\tau_{n}}$ be the zero section of the bundle

$$
\varphi^{*}(E G) \times_{G} E_{n} \longrightarrow X .
$$


Then define

$$
\Omega^{\infty}(E \wedge(\varphi, s))=\operatorname{colim}_{k} \Omega^{k}\left(\varphi^{*}(E G) \times_{G} E_{n+k} /\left(s_{\tau_{n}}(X) \cup\{*\} \times E_{n+k}\right)\right),
$$

where the colimit is taken along the adjoints of maps

$$
\begin{aligned}
\Sigma\left(\varphi^{*}(E G) \times{ }_{G} E_{n} / s_{\tau_{n}}(X) \cup\{*\} \times E_{n}\right) & \\
& \longrightarrow \Sigma_{X}\left(\varphi^{*}(E G) \times{ }_{G} E_{n}\right) /\left(s_{\tau_{n}}(X) \cup\{*\} \times \Sigma E_{n}\right) \\
& \longrightarrow \varphi^{*}(E G) \times_{G} E_{n+1} /\left(s_{\tau_{n}}(X) \cup\{*\} \times E_{n+1}\right) .
\end{aligned}
$$

The above construction gives us a functor

$$
\Omega^{\infty}\left({ }_{\tau} E \wedge(-)\right): \operatorname{Spaces}_{B G} \longrightarrow \text { Spaces }_{*} .
$$

Definition 15. The twisted homology theory associated with an action $\tau$ of a topological group $G$ on a spectrum $E$ is defined to be the composition

$$
{ }_{\tau} \widetilde{E}_{*}(-): \operatorname{Spaces}_{B G} \stackrel{\Omega^{\infty}\left({ }_{\tau} E \wedge(-)\right)}{\longrightarrow} \operatorname{Spaces}_{*} \stackrel{\pi_{*}(-)}{\longrightarrow} \text { Graded Abelian Groups. }
$$

Before we conclude this subsection, let us remark that May and Sigurdsson MS06] developed theory of parametrized spectra which serves as rigorous foundations for twisted homology and cohomology theories.

\subsection{The Atiyah-Segal Twisting of $K$-Theory}

Before we study twistings of Segal's connective $K$-theory in the next section, let us recall the twisting of the complex $K$-theory by Atiyah and Segal AS04 as an example of twisted cohomology theory.

Already in late 1960s, Karoubi [Kar68, DK70] proposed to twist the complex $K$-theory $K^{*}(X)$ and defined $K$-theory with local coefficients in order to establish analogues of the Thom isomorphism and the Poincaré dueality in $K$-theory. J. Rosenberg Ros89] independently introduced a twisted version of $K$-theory in the context of $C^{*}$-algebras. A more general way of twisting $K^{*}(X)$ was discovered by Atiyah and Segal in AS04.

Note that our definition of a twisting of a representable functor depends on an explicit description of a space representing the functor. There are several popular choices for a representing space of the complex $K$-theory. In algebraic topology, we often use $B U \times \mathbb{Z}$

$$
K(X) \cong[X, B U \times \mathbb{Z}]
$$

where $B U$ is a certain colimit of the complex Grassmannian manifolds. Atiyah and Segal chose the space of Fredholm operators.

Theorem 3 (Atiyah). Let $H$ be a separable Hilbert space over $\mathbb{C}$. There is an isomorphism

$$
[X, \operatorname{Fred}(H)] \cong K(X)
$$

for any compact Hausdorff space $X$, where $\operatorname{Fred}(H)$ is the space of Fredholm operators with norm topology. 
We need a topological group $G$ acting on $\operatorname{Fred}(H)$ to twist $[X, \operatorname{Fred}(H)]$. Atiyah and Segal chose $G=P U(H)$, the projective unitary group of $H$. $P U(H)$ acts on $\operatorname{Fred}(H)$ by conjugation

$$
\tau_{A S}: P U(H) \times \operatorname{Fred}(H) \longrightarrow P U(H) .
$$

The Atiyah-Segal twisted $K$-theory $\tau_{A S} K$ is obtained by twisting the representable functor $K(X)=[X, \operatorname{Fred}(H)]$ by this action. Thus it is a functor

$$
\tau_{A S} K:(\text { Spaces } \downarrow B P U(H))^{\mathrm{op}} \longrightarrow \text { Abelian Groups }
$$

defined by

$$
\tau_{A S} K(\varphi)=\pi_{0}\left(\Gamma\left(\varphi^{*}(E P U(H)) \times_{P U(H)} \operatorname{Fred}(\mathrm{H})\right)\right) .
$$

One of the most important fact is that the contractibility of $U(H)$ implies

$$
B P U(H) \simeq K(\mathbb{Z}, 3)
$$

and an object $\varphi: X \rightarrow B P U(H)$ in Spaces $\downarrow B P U(H)$ represents a three dimensional integral cohomology class of $X$ under the isomorphism

$$
[X, B P U(H)] \cong[X, K(\mathbb{Z}, 3)] \cong H^{3}(X ; \mathbb{Z}) .
$$

In order to extend $\tau_{A S} K$ into a generalized cohomology theory satisfying the axioms in Definition[11, we first need a good representing spectrum. One of the choices is the spectrum constructed by Atiyah and Singer in [AS69. They proved that the space of skew-adjoint Fredholm operators $\widehat{\text { Fred }}(H)$ consists of three components, two of which are contractible, and that the remaining component $\operatorname{Fred}_{*}(H)$ represents $K^{-1}(-)$. They also extended their construction by using Clifford algebras and found a spectrum representing the generalized cohomology theory associated with $K$-theory.

In order to use tools and techniques from modern homotopy theory, however, we should represent $K$-theory by a symmetric or an orthogonal spectrum and twist them. A representation of $K$-cohomology theory by an orthogonal spectrum was found by Bunke, Joachim, and Stolz in BJS03. An extension of Atiyah-Segal twisting to $K$-cohomology theory is described by Waldmüller Wal by using the Bunke-Joachim-Stolz specturm.

Since the purpose of the first half of this article is to give an overview of twisted homology and cohomology theories, going into technical details of orthogonal or symmetric spectra is beyond our scope. We refer the paper by Waldmüller for details.

\section{Segal's K-Homology Theory}

In an intriguing paper Seg77], G. Segal found a factorization of the connective $K O$-homology functor $\mathrm{kO}_{*}(-)$ 


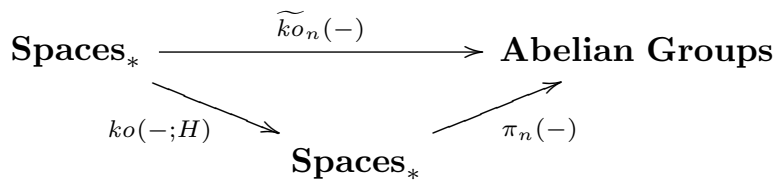

by constructing a space-level functor $k o(-; H)$ 1, where $H$ is an inner product space of countable dimension over $\mathbb{R}$.

The space $k o(X ; H)$ can be described as the space of finite families of finite dimensional vector subspaces of $H$ labelled by points in $X$ which are perpendicular to each other if labelling points are different:

$$
k o(X ; H)=\left\{\begin{array}{l|l}
{\left[V_{x_{1}}, V_{x_{2}}, \cdots, V_{x_{j}}\right]} & \begin{array}{l}
x_{i} \in X \\
V_{x_{i}} \subset H \text { : finite dim. } \\
x_{i} \neq x_{i^{\prime}} \text { if } i \neq i^{\prime} \\
V_{x_{i}} \perp V_{x_{i^{\prime}}} \text { if } i \neq i^{\prime} .
\end{array}
\end{array}\right\}
$$

The definition of $k o(X ; H)$ can be easily modified to give us a complex version of the above diagram

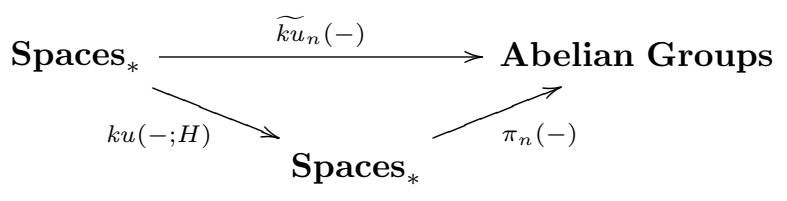

Segal described a way to define a topology on this set and proved that the above diagram is commutative. He also established a way to relate $\pi_{n}(k o(X ; H))$ to $K O_{n}(X)$.

Segal's description of the topology is, however, somewhat obscure. With the above definition, it is not clear how to define a twisted version of $k u(X ; H)$, either. In order to resolve these difficulties, we propose a new definition of the functor $k o(-; H)$ and $k u(-; H)$ and prove basic properties.

Our description allows us to define a twisted version of Segal's $K$-theory. As far as the author knows, there is no known description of a twisted version of Segal's $K$-homology theory. In other words, we extend the above diagram to

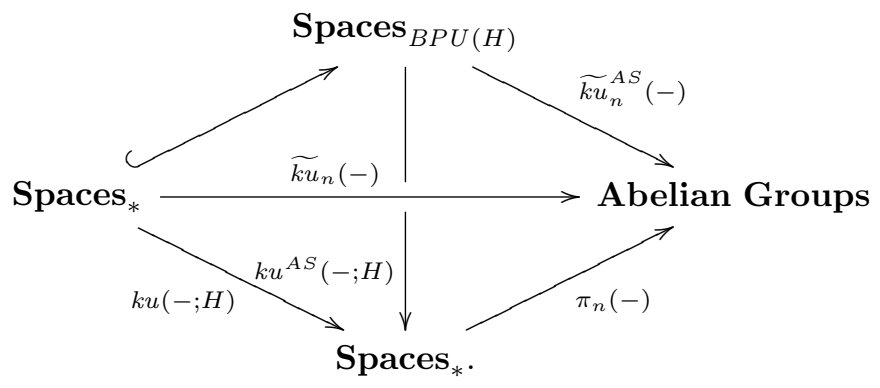

\footnotetext{
${ }^{1}$ Segal used the notation $F(-)$ but we prefer to use $k o(-; H)$ in order to distinguish the real and the complex cases.
} 
Theorem 4. Let Spaces $_{B P U(H)}$ denoted the category of ex-spaces over BPU $(H)$. Then there exists a functor

$$
k u^{A S}: \operatorname{Spaces}_{B P U(H)} \longrightarrow \text { Spaces }_{*},
$$

satisfying the following properties:

1. for a trivial twisting, i.e. a constant map $*_{X}: X \rightarrow * \hookrightarrow B P U(H)$, it agrees with Segal's $k u(X ; H)$

$$
k u^{A S}\left(*_{X}, * ; H\right)=k u(X ; H) ;
$$

2. $k u^{A S}$ is a linear functor, in the sense of Definition 0 Namely it converts a cofibration into a quasifibration.

The second part of this theorem can be obtained as an application of a more general method. One of the most famous examples of linear functors is the infinite symmetric product functor of Dold and Thom DT58.

$$
\mathrm{SP}^{\infty}: \text { Spaces }_{*} \longrightarrow \text { Topological Monoids }
$$

which gives rise to the integral homology groups

$$
\pi_{n}\left(\mathrm{SP}^{\infty}(X)\right) \cong \widetilde{H}_{n}(X ; \mathbb{Z})
$$

Given a diagram

$$
X \stackrel{f}{\longleftarrow} Z \stackrel{g}{\longrightarrow} Y,
$$

let $X \cup_{f} Z \times I \cup_{g} Y$ be the double mapping cylinder. There is a natural homeomorphism

$$
\mathrm{SP}^{\infty}\left(X \cup_{f} Z \times I \cup_{g} Y\right) \cong\left|B_{*}\left(\operatorname{SP}^{\infty}(X), \mathrm{SP}^{\infty}(Z), \mathrm{SP}^{\infty}(Y)\right)\right|,
$$

where $B_{*}(-,-,-)$ is the geometric bar construction for topological monoids. The fact that $\mathrm{SP}^{\infty}$ is linear follows from the observation that the collapsing $Y \rightarrow *$ induces a quasifibration

$$
\left|B_{*}\left(\mathrm{SP}^{\infty}(X), \mathrm{SP}^{\infty}(Z), \mathrm{SP}^{\infty}(Y)\right)\right| \longrightarrow\left|B_{*}\left(\mathrm{SP}^{\infty}(X), \mathrm{SP}^{\infty}(Z), \mathrm{SP}^{\infty}(*)\right)\right| .
$$

Unfortunately our functor $k u^{A S}(\varphi, s ; H)$ does not take values in the category of topological monoids. In order to prove $k u^{A S}(\varphi, s ; H)$ is linear by using this idea, we use the following theorem proved in a separate paper Tam.

Theorem 5. (Tam], Theorem 1) Let $M$ be a partial topological monoid with a good unit acting on $X$ and $Y$ from the right and the left, respectively. If the inclusions

$$
\begin{aligned}
B_{n}(X, M, Y) & \hookrightarrow X \times M^{n} \times Y \\
B_{n}(X, M, *) & \hookrightarrow X \times M^{n} \\
C_{M}(Y) & \hookrightarrow M \times Y
\end{aligned}
$$


are weak equivalences for each $n$ and if the action of $m \in M$ on $Y$ induces a weak equivalence

$$
Y_{m}=\left\{y \in Y \mid(m, y) \in C_{M}(Y)\right\} \stackrel{m \cdot}{\longrightarrow} Y
$$

for each $m \in M$, then

$$
p^{Y}:\left|B_{*}(X, M, Y)\right| \longrightarrow\left|B_{*}(X, M, *)\right|
$$

is a quasifibration.

Notations in the above theorem will be explained in 3.2

\subsection{Segal's $K$-Homology Theory by Projective Space Bundles}

Let us first recall Segal's original construction of connective $K$-homology theory introduced in Seg77. For a compact Hausdorff space $X$, Segal considers the following functor

$$
M_{n}(X ; \mathbb{R})=\operatorname{Hom}_{\mathrm{alg}}\left(C(X ; \mathbb{R}), M_{n}(\mathbb{R})\right),
$$

where $C(X ; \mathbb{R})$ is the Banach algebra of continuous real-valued functions on $X$ and $\operatorname{Hom}_{\text {alg }}(-,-)$ denotes the space of bounded algebra homomorphisms. Since we are interested in the twisted $K$-theory, let us consider the complex version, i.e.

$$
M\left(X ; \mathbb{C}^{n}\right)=\operatorname{Hom}_{*-\operatorname{alg}}\left(C(X), M_{n}(\mathbb{C})\right),
$$

where $C(X)$ is the $C^{*}$-algebra of continuous complex-valued functions on $X$.

Since $C(X)$ is commutative, its image under $\varphi \in \operatorname{Hom}_{*-a l g}\left(C(X), M_{n}(\mathbb{C})\right)$ is a finite dimensional commutative subalgebra of $M_{n}(\mathbb{C})$ consisting of normal matrices. Thus $\varphi(C(X))$ is simultaneously diagonalizable by a unitary matrix, i.e. there exists a unitary matrix $A \in U(n)$ with

$$
A^{-1} \varphi(f) A=\left(\begin{array}{cccc}
\lambda_{1}(f) & 0 & \cdots & 0 \\
0 & \lambda_{2}(f) & \ddots & \vdots \\
\vdots & \ddots & \ddots & 0 \\
0 & \cdots & 0 & \lambda_{n}(f)
\end{array}\right)
$$

for $f \in C(X)$. The ordering of $\lambda_{i}$ 's depends on the choice of $A$. Let $\lambda_{i_{1}}(f), \cdots, \lambda_{i_{k}}(f)$ be distinct eigenvalues of $\varphi(f)$. Then it is a basic fact in linear algebra that $\varphi(f)$ can be recovered from these distinct eigenvalues and the corresponding eigenspace decomposition

$$
\mathbb{C}^{n}=V_{1} \oplus \cdots \oplus V_{k} .
$$

Each $\lambda_{i_{\ell}}$ is a $C^{*}$-algebra homomorphism

$$
\lambda_{i_{\ell}}: C(X) \longrightarrow \mathbb{C}
$$

and thus can be identified with a point in $X$ under the famous Gel'fandNaimark duality. 
Theorem 6 (Gel'fand-Naimark duality). The functor

\section{$C:(\text { Compact Hausdorff })^{\mathrm{op}} \longrightarrow$ Commutative $C^{*}$-Algebras}

is a contravariant equivalence of categories.

Proof. See, for example, [DB86].

Thus the set $M_{n}(X ; \mathbb{C})$ is in one-to-one correspondence with the set

$$
\coprod_{k=1}^{n}\left\{\begin{array}{l|l}
\left(V_{1}, \cdots, V_{k} ; x_{1}, \cdots, x_{k}\right) & \begin{array}{l}
V_{1}+\cdots+V_{k}=\mathbb{C}^{n} \\
V_{\ell} \perp V_{\ell^{\prime}}, \text { if } \ell \neq \ell^{\prime} \\
x_{1}, \cdots, x_{k} \in X \\
x_{\ell} \neq x_{\ell^{\prime}}, \text { if } \ell \neq \ell^{\prime}
\end{array}
\end{array}\right\} / \Sigma_{k} .
$$

The above definition of $M_{n}(X ; \mathbb{C})$ is slightly different from Segal's. Segal uses the algebra of functions $C_{0}(X)$ vanishing at the base point $x_{0}$ instead of $C(X)$ and then use the base point to take the colimit

$$
k u(X)=\operatorname{colim}_{n \rightarrow \infty} M_{n}(X ; \mathbb{C}) .
$$

With our $M_{n}(X ; \mathbb{C})$, the following construction is equivalent to Segal's. For an infinite dimensional complex inner product space $H$ with a countable basis, consider the following set

$M^{(k)}(X ; H)=\left\{\begin{array}{l|l}\left(V_{1}, \cdots, V_{k} ; x_{1}, \cdots, x_{k}\right) & \begin{array}{l}V_{1}, \cdots, V_{k} \subset U \text { (finite dim. subspaces) } \\ V_{\ell} \perp V_{\ell^{\prime}} \text { if } \ell \neq \ell^{\prime} \\ x_{1}, \cdots, x_{k} \in X \\ x_{\ell} \neq x_{\ell^{\prime}} \text { if } \ell \neq \ell^{\prime}\end{array}\end{array} \mid\right.$.

When $X$ has a base point $*$, we may glue these spaces together to form

$$
k u(X ; H)=\left(\coprod_{k} M^{(k)}(X ; H) / \Sigma_{k}\right) / \sim
$$

where

$\left[V_{1}, \cdots, V_{k} ; x_{1}, \cdots, x_{k}\right] \sim\left[V_{1}, \cdots, V_{i-1}, V_{i+1}, \cdots, V_{k} ; x_{1}, \cdots, x_{i-1}, x_{i+1}, \cdots, x_{k}\right]$

if $x_{i}=*$. Then Segal's construction is nothing but $k u\left(X ; \mathbb{C}^{\infty}\right)$, where

$$
\mathbb{C}^{\infty}=\operatorname{colim}_{n} \mathbb{C}^{n}
$$

Since the ordinary homology group $\widetilde{H}_{*}(X ; \mathbb{Z})$ can be described as

$$
\widetilde{H}_{n}(X ; \mathbb{Z})=\pi_{n}\left(\mathrm{SP}^{\infty}(X)\right)
$$

for a reasonably good space $X$ by using the infinite symmetric product DT58. 


$$
\mathrm{SP}^{\infty}(X)=\left\{\begin{array}{l|l}
{\left[n_{1}, \cdots, n_{k} ; x_{1}, \cdots, x_{k}\right]} & \begin{array}{l}
n_{1}, \cdots, n_{k} \in \mathbb{N} \\
x_{1}, \cdots, x_{k} \in X \\
x_{\ell} \neq x_{\ell^{\prime}} \text { if } \ell \neq \ell^{\prime}
\end{array}
\end{array}\right\}
$$

the definition of $k u(X ; H)$ looks reasonable. It is obtained by the standard process of defining $K$-theory, i.e. by replacing natural numbers by finite dimensional vector spaces. If we write an element of $\operatorname{SP}^{\infty}(X)$ as a formal sum

$$
n_{1} x_{1}+n_{2} x_{2}+\cdots+n_{k} x_{k}
$$

elements of $k u(X ; H)$ are obtained by taking a "categorification" of coefficients. See BD98 for an exposition of categorification.

However, it is not clear how to put a reasonable topology on this set. Segal gives a brief description of a topology to be defined. He required the following two conditions:

1. Distinct points $x_{i}$ and $x_{j}$ in $\left[V_{1}, \cdots, V_{k} ; x_{1}, \cdots, x_{k}\right]$ can move into coincidence at $x_{\ell}^{\prime}$ in $\left[V_{1}^{\prime}, \cdots, V_{k-1}^{\prime} ; x_{1}^{\prime}, \cdots, x_{k-1}^{\prime}\right]$, and then in the limit

$$
V_{\ell}^{\prime}=V_{i} \oplus V_{j}
$$

2. A point $x_{i}$ in $\left[V_{1}, \cdots, V_{k} ; x_{1}, \cdots, x_{k}\right]$ can move to the base point $*$, and then $V_{i}$ and $x_{i}$ are removed in the limit.

In order to define a twisted version, we need to be more precise. Note that the second condition is our base point relation in the definition of $k u(X ; H)$. In order to define a topology satisfying Segal's first condition, we need to use the direct sum operation. In the case of the infinite symmetric product, the coefficient set $\mathbb{N}$ has the discrete topology and the addition is continuous.

The space $k u(X ; H)$ is defined by using finite dimensional subspaces in $H$. Thus it seems natural to describe elements in $k u(X ; H)$ by using points in the Grassmannian manifold of finite dimensional subspaces in $H$. However, the orthogonality condition on the subspaces in the coefficients of an element of $k u(X ; H)$ depends on points in $X$. In order to make the condition more precise, let us enlarge the Grassmannian manifolds by using the linear isometries operad.

Definition 16. Let $H$ ba a complex inner product space with a countable basis. Define

$$
\mathcal{L}(0 ; H)=\{*\}
$$

and for $j \geq 1$, define

$$
\mathcal{L}(j ; H)=\operatorname{Iso}(\underbrace{H \oplus \cdots \oplus H}_{j}, H)
$$

with the norm topology, where Iso denotes the set of linear isometries. 
Proposition 4. When $H$ is infinite dimensional, each $\mathcal{L}(j ; H)$ is contractible and $\mathcal{L}(H)=\{\mathcal{L}(j ; H)\}_{j \geq 0}$ forms an operad under composition. Thus $\mathcal{L}(H)$ is an $E_{\infty}$-operad.

The operad $\mathcal{L}(H)$ is called the linear isometries operad based on $H$. When $H$ is clear from the context, we simply denote it by $\mathcal{L}$. The linear isometries operad $\mathcal{L}$ plays an essential role in the construction of the symmetric monoidal category of coordinate free spectra by Elmendorf, Kriz, Mandell, and May. See their book EKMM97 for basic properties of the linear isometries operad $\mathcal{L}$, including a proof of the above Proposition.

Note that $\mathcal{L}(1 ; H)$ is the group of unitary operators $U(H)$ on $H$ if $H$ is a Hilbert space. (In general, $\mathcal{C}(1)$ is a monoid for any operad $\mathcal{C}$.) From now on, we fix a separable Hilbert space $H$ over $\mathbb{C}$.

We reconstruct $k u(X ; H)$ by gluing projective spaces via the action of $\mathcal{L}(H)$.

Definition 17. Let $\mathbb{P}(H)$ be the space of lines in $H$ through the origin,

$$
\mathbb{P}(H)=\{\ell \subset H \mid \operatorname{dim} \ell=1\} .
$$

$\mathbb{P}(H)$ has a natural action of $\mathcal{L}(1 ; H)$. In order to obtain higher dimensional subspaces, we take products of $\mathbb{P}(H)$ 's over $\mathcal{L}(H)$.

Definition 18. For $\mathcal{L}(1 ; H)$-spaces, $X$ and $Y$, define

$$
X \times_{\mathcal{L}(H)} Y=\mathcal{L}(2 ; H) \times_{\mathcal{L}(1 ; H) \times \mathcal{L}(1 ; H)}(X \times Y) .
$$

$X \times_{\mathcal{L}(H)} Y$ is the quotient space of $\mathcal{L}(2 ; H) \times X \times Y$ under the relation

$$
\left(\varphi \circ\left(f_{1} \oplus f_{2}\right) ; x, y\right) \sim\left(\varphi ; f_{1}(x), f_{2}(y)\right) .
$$

An important fact is this "product" is associative.

Lemma 3. For $\mathcal{L}(1 ; H)$-spaces, $X, Y$, and $Z$, we have the following natural homeomorphisms

$$
\begin{aligned}
\left(X \times_{\mathcal{L}(H)} Y\right) \times_{\mathcal{L}(H)} Z & \cong \mathcal{L}(3 ; H) \times_{\mathcal{L}(1 ; H)^{3}}(X \times Y \times Z) \\
& \cong X \times_{\mathcal{L}(H)}\left(Y \times_{\mathcal{L}(H)} Z\right) .
\end{aligned}
$$

Proof. See EKMM97.

Definition 19. Define

$$
\mathbb{P}_{0}(H)=\{*\}
$$

and, for $j \geq 1$, define

$$
\mathbb{P}_{j}(H)=\underbrace{\mathbb{P}(H) \times_{\mathcal{L}(H)} \cdots \times_{\mathcal{L}(H)} \mathbb{P}(H)}_{j} \cong \mathcal{L}(j ; H) \times_{\mathcal{L}(1 ; H)^{j}} \mathbb{P}(H)^{j} .
$$


Now we are ready to give a precise definition of $k u(X ; H)$.

Definition 20. For a based space $X$, define relations $\underset{*}{\sim}$ and $\underset{G r}{\sim}$ on the disjoint union $\coprod_{j=0}^{\infty} \mathbb{P}_{j}(H) \times_{\Sigma_{j}} X^{j}$ as follows:

1. The relation $\underset{*}{\sim}$ is the base point relation, i.e.

$$
\begin{aligned}
{\left[\varphi, \ell_{1}, \cdots, \ell_{j} ; x_{1}, \cdots, x_{j}\right] \underset{*}{\sim} } & \\
& {\left[s_{i}(\varphi), \ell_{1}, \cdots, \ell_{i-1}, \ell_{i+1}, \cdots, \ell_{j} ; x_{1}, \cdots, x_{i-1}, x_{i+1}, \cdots, x_{j}\right] }
\end{aligned}
$$

if $x_{i}=*$, where $s_{i}$ is defined by the operad structure

$$
\mathcal{L}(j) \cong \mathcal{L}(j) \times \mathcal{L}(0) \stackrel{\circ_{i}}{\longrightarrow} \mathcal{L}(j-1) .
$$

2. The relation $\underset{G r}{\sim}$ is defined as follows: for elements in the nondegenerate form (all base points removed)

$$
\left[\varphi ; \ell_{1}, \cdots, \ell_{j} ; x_{1}, \cdots, x_{j}\right] \underset{G r}{\underset{G r}{\sim}}\left[\varphi^{\prime} ; \ell_{1}^{\prime}, \cdots, \ell_{j}^{\prime} ; x_{1}^{\prime}, \cdots, x_{j}^{\prime}\right]
$$

if, after a suitable reordering,

a) $x_{i}=x_{i}^{\prime}$ for all $i$

b) $\varphi\left(\ell_{i_{1}} \oplus \cdots \oplus \ell_{i_{k}}\right)=\varphi^{\prime}\left(\ell_{i_{1}}^{\prime} \oplus \cdots \oplus \ell_{i_{k}}^{\prime}\right)$ if $x_{i_{1}}=\cdots=x_{i_{k}}$

Now define

$$
k u(X ; H)=\left(\coprod_{j=0}^{\infty} \mathbb{P}_{j}(H) \times_{\Sigma_{j}} X^{j}\right) /_{*} \underset{*}{\sim} \underset{G r}{.}
$$

The above definition coincides with Segal's definition when $U=\mathbb{C}^{\infty}$. Segal gives a proof of the following theorem by mimicing the proof of a theorem of Dold and Thom DT58.

Theorem 7 (Segal). If $(X, A)$ is an $N D R$ pair and $\left(A, a_{0}\right)$ is a strong $N D R$ pair, then the following sequence is a quasifibration

$$
k u(A ; H) \longrightarrow k u(X ; H) \longrightarrow k u(X / A ; H) .
$$

We give an alternative proof and a proof of a twisted version of this theorem in 3.2 .

In the rest of this subsection, we concentrate on constructing a twisted version of Segal's $K$-homology theory as a functor

$$
k u^{A S}: \operatorname{Spaces}_{B P U(H)} \longrightarrow \text { Spaces }_{*} \cdot
$$

The idea is simple. Note that the definition of the untwisted version can be written as 


$$
\begin{aligned}
& k u(X ; H)=\left.\left(\coprod_{j=0}^{\infty} \mathbb{P}_{j}(H) \times_{\Sigma_{j}} X^{j}\right)\right|_{* \underset{\sim}{\sim} \widetilde{G r}} \\
& =\left.\left(\coprod_{j=0}^{\infty}\left(\mathcal{L}(j ; H) \times_{\mathcal{L}(1 ; H)^{j}}(\mathbb{P}(H) \times X)^{j}\right) / \Sigma_{j}\right)\right|_{\sim}, \underset{\mathrm{Gr}^{\prime}}{ } .
\end{aligned}
$$

In other words, $k u(X ; H)$ is obtained by gluing copies of the trivial $\mathbb{P}(H)$ bundle over $X$ by the action of the linear isometries operad $\mathcal{L}(H)$.

In view of the appearance of $\mathbb{P}(H)$-bundles in the work of Atiyah and Segal [AS04, it is natural to replace the trivial bundle with the $\mathbb{P}(H)$-bundle induced by a map $\varphi: X \rightarrow B P U(H)$. We need to be a little bit careful to obtain a fiberwise $\mathcal{L}(1 ; H)$-action.

Lemma 4. Define an action

$$
\mathcal{L}(1 ; H) \times P U(H) \longrightarrow P U(H)
$$

by conjugation. Then this action respects the group structure on $P U(H)$.

Recall from Theorem 2 that we regard the universal bundles as a functor on the category of topological groups.

Corollary 2. The projection of the universal bundle

$$
E P U(H) \longrightarrow B P U(H)
$$

respects the action of $\mathcal{L}(1 ; H)$. Thus we obtain an action of $\mathcal{L}(1 ; H)$ on the associated $\mathbb{P}(H)$-bundle

$$
E \mathbb{P}(H)=E P U(H) \times_{P U(H)} \mathbb{P}(H) \longrightarrow B P U(H) .
$$

The action on the total space is given by

$$
f \cdot[e, a]=\left[f e f^{-1}, f a\right] .
$$

Note that we need to consider actions of $\mathcal{L}(1 ; H)$ not only on $E \mathbb{P}(H)$ but also on $B P U(H)$. The following fact guarantees we can work in the category of $\mathcal{L}(1 ; H)$-spaces.

Lemma 5. Let $\mathcal{L} \mathbf{S p a c e s}_{*}$ be the category of based spaces with $\mathcal{L}(1 ; H)$ actions. Then the functor

$$
\mathcal{L}: \operatorname{Spaces}_{*} \longrightarrow \mathcal{L} \operatorname{Spaces}_{*}
$$

defined by

$$
\mathcal{L}(X)=\mathcal{L}(1 ; H) \wedge X_{+}
$$

induces an equivalence of homotopy categories. 
Proof. See [EKMM97, where an analogous fact for spectra is proved. It is straightforward to modify their argument to based spaces.

In the rest of this subsection, we assume all spaces and maps belong to the category $\mathcal{L} \operatorname{Spaces}_{*}$. In particular, for a map

$$
\varphi: X \longrightarrow B P U(H)
$$

in $\mathcal{L}$ Spaces $_{*}$, the associated $\mathbb{P}(H)$-bundle

$$
p_{\varphi}: E_{\varphi}(\mathbb{P}(H)) \longrightarrow X
$$

is equipped with an action of $\mathcal{L}(1 ; H)$. Note that we have a map

$$
\mathcal{L}(j ; H) \times_{\mathcal{L}(1 ; H)^{j}} E_{\varphi}(\mathbb{P}(H))^{j} \longrightarrow E_{\varphi}\left(\operatorname{Gr}_{j}(H)\right),
$$

where $\operatorname{Gr}_{j}(H)$ is the space of $j$-dimensional subspaces in $H$ and $E_{\varphi}\left(\operatorname{Gr}_{j}(H)\right)$ is the $\operatorname{Gr}_{j}(H)$-bundle associated with $E_{\varphi}=\varphi^{*}(E P U(H))$.

The following is our definition of $k u^{A S}(-; H)$.

Definition 21. For an object $(\varphi, s)$ in $\operatorname{Spaces}_{B P U(H)}$ with $\varphi: X \rightarrow B P U(H)$, define

$$
k u^{A S}(\varphi, s ; H)=\left.\left(\coprod_{j=0}^{\infty}\left(\mathcal{L}(j ; H) \times_{\mathcal{L}(1 ; H)^{j}} E_{\varphi}^{j}\right) / \Sigma_{j}\right)\right|_{\sim} \underset{*}{\sim} \cdot
$$

where the relations $\underset{*}{\sim} \underset{\text { Gr }}{\widetilde{T}}$ are equivalence relations generated by the following relations:

1. For $\left[f ; e_{1}, \cdots, e_{j}\right]$, if $e_{i} \in s(B P U(H))$,

$$
\left[f ; e_{1}, \cdots, e_{j}\right] \sim\left[s_{i}(f) ; e_{1}, \cdots, e_{i-1}, e_{i+1}, \cdots e_{j}\right] .
$$

2. Suppose none of $e_{i}$ 's and $e_{i}^{\prime}$ 's in $\left[f ; e_{1}, \cdots, e_{j}\right],\left[f^{\prime}: e_{1}^{\prime}, \cdots, e_{j}^{\prime}\right]$ belong to $s(B P U(H))$. Then

$$
\left[f ; e_{1}, \cdots, e_{j}\right] \underset{G r}{\widetilde{T}}\left[f^{\prime}: e_{1}^{\prime}, \cdots, e_{j}^{\prime}\right]
$$

if and only if the following conditions hold:

a) We may rearrange $e_{i}$ 's and $e_{i}^{\prime}$ 's under the action of $\Sigma_{j}$ so that

$$
p_{\varphi}\left(e_{i}\right)=p_{\varphi}\left(e_{i}^{\prime}\right)
$$

for all $i$; 
b) Under the above condition, write

$$
\begin{aligned}
& \left(p_{\varphi}\left(e_{1}\right), \cdots, p_{\varphi}\left(e_{j}\right)\right)=\left(p_{\varphi}\left(e_{1}^{\prime}\right), \cdots, p_{\varphi}\left(e_{j}^{\prime}\right)\right)=\left(\Delta^{k_{1}}\left(x_{1}\right), \cdots, \Delta^{k_{m}}\left(x_{m}\right)\right) \\
& \text { with } x_{i} \neq x_{i^{\prime}} \text { if } i \neq i^{\prime} \text {. For } k_{1}+\cdots+k_{i-1}+1 \leq m \leq k_{1}+\cdots+k_{i}, \\
& \text { write } \\
& \qquad \begin{array}{l}
e_{m}=\left(x_{i}, u_{m}\right) \\
e_{m}^{\prime}=\left(x_{i}, u_{m}^{\prime}\right)
\end{array}
\end{aligned}
$$

where $u_{m}, u_{m}^{\prime}$ are elements of the total space of the universal $\mathbb{P}(H)$ bundle over $B P U(H)$. Then

$$
f\left(0 \oplus\left\langle u_{m} \mid m\right\rangle \oplus 0\right)=f^{\prime}\left(0 \oplus\left\langle u_{m}^{\prime} \mid m\right\rangle \oplus 0\right),
$$

where $m$ ranges over $k_{1}+\cdots+k_{i-1}+1 \leq m \leq k_{1}+\cdots+k_{i}$ and we regard $f, f^{\prime}$ as maps

$$
f, f^{\prime}: E_{\varphi}(\mathbb{P}(H))^{j} \longrightarrow E_{\varphi}\left(\operatorname{Gr}_{j}(H)\right) .
$$

The twisted connective K-homology $\widetilde{k u_{*}}{ }^{A S}(-)$ is defined by

$$
\widetilde{k u}_{*}^{A S}(\varphi, s ; H)=\pi_{*}\left(k u^{A S}(\varphi, s ; H)\right) .
$$

("AS" stands for "Atiyah-Segal twisting".)

If $(\varphi, s)$ comes from a based space $X$, i.e.

$$
\varphi=* \vee 1_{B P U(H)}: X \vee B P U(H) \longrightarrow B P U(H),
$$

the action of $\mathcal{L}(1 ; H)$ on $E_{\varphi}(\mathbb{P}(H))$ reduces to the action of $\mathcal{L}(1 ; H)$ on $\mathbb{P}(H)$ and we have an identification

$$
k u^{A S}(\varphi, s ; H) \cong k u(X ; H) .
$$

Thus $k u^{A S}(-; H)$ is an extension of Segal's construction. In order to prove that $\widetilde{k u_{*}}{ }_{*}(-)$ is a homology theory on $\mathbf{S p a c e s}_{B P U(H)}$, we use generalized two-sided bar constructions.

\subsection{The Linearity of Segal's $K$-Homology Theory}

Let us consider the untwisted case first. In order to prove that the functor $k u(-; H)$ is linear, we need to show that $k u(-; H)$ converts a wedge sum into a product up to a weak equivalence and a cofibration into a quasifibration. It was Segal who first realized that the second property is essentially a consequence of the first property and introduced the notion of $\Gamma$-space Seg74. 
Later Woolfson developed Segal's idea further whose result was used by Shimakawa [Shi01, Shi07] to prove certain functors constructed from configuration spaces are linear.

Our approach is also based on Segal's idea but makes an explicit use of the two-sided bar construction, which can be also used to prove the linearity of our twisted version of the connective K-homology theory.

Let us first recall the two-sided bar construction for topological monoids.

Definition 22. Let $G$ be a topological monoid and

$$
\begin{aligned}
& X \times G \longrightarrow X \\
& G \times Y \longrightarrow Y
\end{aligned}
$$

be right and left $G$ actions.

For $n \geq 0$, define

$$
B_{n}(X, G, Y)=X \times G^{n} \times Y
$$

and, for $0 \leq i \leq n$, define

$$
\begin{aligned}
d_{i} & : B_{n}(X, G, Y) \longrightarrow B_{n-1}(X, G, Y) \\
s_{i} & : B_{n}(X, G, Y) \longrightarrow B_{n+1}(X, G, Y)
\end{aligned}
$$

by

$$
\begin{aligned}
d_{0}\left(x ; g_{1}, \cdots, g_{n} ; y\right) & =\left(x g_{1}, g_{2}, \cdots, g_{n} ; y\right) \\
d_{i}\left(x, g_{1}, \cdots, g_{n} ; y\right) & =\left(x, g_{1}, \cdots, g_{i} g_{i+1}, \cdots, g_{n} ; y\right) \\
d_{n}\left(x, g_{1}, \cdots, g_{n} ; y\right) & =\left(x, g_{1}, \cdots, g_{n-1}, g_{n} y\right) \\
s_{i}\left(x ; g_{1}, \cdots, g_{n} ; y\right) & =\left(x ; g_{1}, \cdots, g_{i}, e, g_{i+1}, \cdots, g_{n} ; y\right)
\end{aligned}
$$

where $e \in G$ is the unit.

$B_{*}(X, G, Y)=\left\{B_{n}(X, G, Y), d_{i}, s_{i}\right\}$ is called the two-sided bar construction.

$B_{*}(X, G, Y)$ has a structure of a simplicial space. We denote the geometric realization of a simplicial space $X_{*}$ by $\left|X_{*}\right|$. by

Recall that the infinite symmetric product of a pointed space $X$ is defined

$$
\operatorname{SP}^{\infty}(X)=\left(\coprod_{j} X^{j} / \Sigma_{j}\right) / \sim
$$

where the relation $\sim$ is defined by

$$
\left[x_{1}, \cdots, x_{j}\right] \sim\left[x_{1}, \cdots, x_{i-1}, x_{i+1}, \cdots, x_{j}\right]
$$

if $x_{i}=*$. Our idea is based on the following obsevation. 
Proposition 5. Given maps

$$
X \stackrel{f}{\longleftarrow} Y \stackrel{g}{\longrightarrow} Z,
$$

we have the following homeomorphism

$$
\mathrm{SP}^{\infty}\left(M_{f, g}\right) \cong\left|B\left(\mathrm{SP}^{\infty}(X), \mathrm{SP}^{\infty}(Y), \mathrm{SP}^{\infty}(Z)\right)\right|,
$$

where

$$
M_{f, g}=(X \amalg(Y \times I) \amalg Z) /(y, 0) \sim f(y),(y, 1) \sim g(y)
$$

is the double mapping cylinder.

Proof. Note that the standard $n$-simplex can be described as

$$
\Delta^{n}=\left\{\left(t_{1}, \cdots, t_{n}\right) \in \mathbb{R}^{n} \mid 0 \leq t_{1} \leq \cdots \leq t_{n} \leq 1\right\} \cong \operatorname{SP}^{n}([0,1]) .
$$

By arranging elements of $Y \times I$ in $M_{f, g}$ in the increasing order of elements in $I$, we have a desired homeomorphism.

The well-known theorem of Dold and Thom saying that $\mathrm{SP}^{\infty}$ is linear is an easy consequence of the following basic properties of the two-sided bar construction.

Proposition 6. For any space $X$, we have homotopy equivalences

$$
|B(G, G, X)| \simeq X \simeq|B(X, G, G)| .
$$

In particular, $|B(G, G, *)|$ and $|B(*, G, G)|$ are contractible.

Theorem 8 ([Mil67, May75]). Let $G$ be a topological monoid acting on $X$ and $Y$ from the right and the left, respectively. If $(G,\{e\})$ is a strong NDR pair and $\pi_{0}(G)$ is a group, then the following sequence is a quasifibration

$$
Y \longrightarrow\left|B_{*}(X, G, Y)\right| \stackrel{p}{\longrightarrow}\left|B_{*}(X, G, *)\right|,
$$

where $p$ is given by collapsing $Y$ to a single point.

By comparing with the quasifibration in Theorem 8 , we have the following.

Corollary 3. If $Y$ is path-connected and $\left(Y, y_{0}\right)$ is a strong NDR pair, then the following sequence is a quasifibration

$$
\mathrm{SP}^{\infty}(Z) \longrightarrow \mathrm{SP}^{\infty}\left(M_{f, g}\right) \longrightarrow \mathrm{SP}^{\infty}\left(X \cup_{f} C Y\right)
$$

Now a theorem of Dold-Thom follows immediately.

Corollary 4 (Dold-Thom). If $(X, A)$ is an NDR pair and $\left(A, a_{0}\right)$ is a strong $N D R$ pair, then the following sequence is a quasifibration

$$
\mathrm{SP}^{\infty}(A) \longrightarrow \mathrm{SP}^{\infty}(X) \longrightarrow \mathrm{SP}^{\infty}(X / A)
$$


Proof. Since $(X, A)$ is an NDR pair, the inclusion

$$
A \hookrightarrow X
$$

is a cofibration and thus its cofiber and the homotopy cofiber have the same homotopy type

$$
X / A \simeq X \cup C A .
$$

Note that

$$
X \simeq X \cup A \times I
$$

and we have a quasifibration

$$
\mathrm{SP}^{\infty}(A) \longrightarrow \mathrm{SP}^{\infty}(X \cup A \times I) \longrightarrow \mathrm{SP}^{\infty}(X \cup C A)
$$

by the above Corollary. Thus we obtain a quasifibration

$$
\mathrm{SP}^{\infty}(A) \longrightarrow \mathrm{SP}^{\infty}(X) \longrightarrow \mathrm{SP}^{\infty}(X / A),
$$

since $\mathrm{SP}^{\infty}$ is a homotopy functor.

It is natural to expect that we can prove the linearity of $k u(-; H)$ by modifying the above proof, since $k u(-; H)$ is obtained from $\mathrm{SP}^{\infty}(-)$ by categorifying natural numbers to vector spaces.

Note that there is a big difference between $\mathrm{SP}^{\infty}(X)$ and $k u(X ; H)$ : $\mathrm{SP}^{\infty}(X)$ is a monoid, while $k u(X ; H)$ is not. We cannot simply concatenate elements in $k u(X ; H)$ because of the orthogonality condition on the subspaces. Any element $\varphi \in \mathcal{L}(2 ; H)=\operatorname{Iso}(H \oplus H, H)$ induces a map

$$
k u(X ; H) \times k u(X ; H) \longrightarrow k u(X ; H \oplus H) \stackrel{\varphi_{*}}{\longrightarrow} k u(X ; H) .
$$

However, there is no way to expect this gives a monoid structure on $k u(X ; H)$. Thus the two-sided bar construction can not be applied to $k u(X ; H)$.

This is the same difficulty we encounter when we try to define a symmetric monoidal structure on the category of spectra under the smash product. An important idea by Elmendorf, Kriz, Mandell, and May to overcome this difficulty is to collect all such products. In our case, we have

$$
\begin{aligned}
k u(X ; H) \times_{\mathcal{L}(H)} & k u(X ; H)= \\
& \mathcal{L}(2 ; H) \times_{\mathcal{L}(1 ; H)^{2}}(k u(X ; H) \times k u(X ; H)) \longrightarrow k u(X ; H) .
\end{aligned}
$$

Thus a natural idea is to replace $\times$ by $\times_{\mathcal{L}(H)}$ in the two-sided bar construction and perform the same construction. We are not going to pursue this idea in this paper. Instead of enlarging the product, the author thinks that a natural way is to restrict our attension to a subspace of a product.

Recall that an element of $k u(X ; H)$ can be represented by a sequence

$$
(\boldsymbol{V}, \boldsymbol{x})=\left(V_{1}, \cdots, V_{j} ; x_{1}, \cdots, x_{j}\right),
$$

where $V_{i}$ 's are finite dimensional subspaces of $H$ with $V_{i} \perp V_{k}, x_{i} \neq x_{k}$ if $i \neq k$, and $x_{i} \neq *$ for all $i$. 
Definition 23. For a pointed space $X$, define the subspace

$$
k u(X ; H) \stackrel{\perp}{\times} k u(Y ; H) \subset k u(X ; H) \times k u(Y ; H)
$$

as follows: for $([\boldsymbol{V} ; \boldsymbol{x}],[\boldsymbol{W} ; \boldsymbol{y}]) \in k u(X ; H) \times k u(X ; H)$ with

$$
\begin{aligned}
(\boldsymbol{V} ; \boldsymbol{x}) & =\left(V_{1}, \cdots, V_{j} ; x_{1}, \cdots, x_{j}\right) \\
(\boldsymbol{W} ; \boldsymbol{y}) & =\left(W_{1}, \cdots, W_{k} ; y_{1}, \cdots, y_{k}\right),
\end{aligned}
$$

define

$$
([\boldsymbol{V} ; \boldsymbol{x}],[\boldsymbol{W} ; \boldsymbol{y}]) \in k u(X ; H) \stackrel{\perp}{\times} k u(X ; H) \Longleftrightarrow V_{i} \perp W_{k} \text { for all } i, k .
$$

Then the concatenation induces a well-defined map

$$
k u(X ; H) \stackrel{\perp}{\times} k u(X ; H) \longrightarrow k u(X ; H) .
$$

Note that $\stackrel{\perp}{\times}$ is associative

$$
(k u(X ; H) \stackrel{\perp}{\times} k u(Y ; H)) \stackrel{\perp}{\times} k u(Z ; H)=k u(X ; H) \stackrel{\perp}{\times}(k u(Y ; H) \stackrel{\perp}{\times} k u(Z ; H)) .
$$

We denote the $k$-fold $\stackrel{\perp}{\times}$-product of $k u(X ; H)$ by $k u(X ; H)^{\stackrel{\perp}{\times} n}$.

Thus we have an analogue of the two-sided bar construction.

Definition 24. Given continuous maps

$$
X \stackrel{f}{\longleftarrow} Y \stackrel{g}{\longrightarrow} Z
$$

define

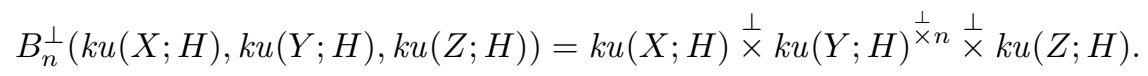

Then we obtain a simplicial space

$B_{*}^{\perp}(k u(X ; H), k u(Y ; H), k u(Z ; H))=\left\{B_{n}^{\perp}(k u(X ; H), k u(Y ; H), k u(Z ; H))\right\}_{n \geq 0}$.

The following identification is analogous to the case of $\mathrm{SP}^{\infty}$.

Lemma 6. Given continuous maps

$$
X \stackrel{f}{\longleftarrow} Y \stackrel{g}{\longrightarrow} Z
$$

we have the following natural homeomorphism

$$
k u\left(M_{f, g} ; H\right) \cong\left|B_{*}^{\perp}(k u(X ; H), k u(Y ; H), k u(Z ; H))\right| .
$$


Proof. Any element of $k u\left(M_{f, g} ; H\right)$ can be written as

$$
\left[\boldsymbol{V}, \boldsymbol{W}_{1}, \cdots, \boldsymbol{W}_{j}, \boldsymbol{U}, \boldsymbol{x},\left(t_{1}, \boldsymbol{y}_{1}\right), \cdots,\left(t_{j}, \boldsymbol{y}_{j}\right), \boldsymbol{z}\right]
$$

where $\boldsymbol{x} \in X^{i}, \boldsymbol{y}_{\ell} \in Y^{j_{\ell}}, 0 \leq t_{1}<\cdots<t_{j} \leq 1$, and $\boldsymbol{z} \in Y^{k}$. Vector spaces $V_{i}, W_{j \ell}, U_{k}$ appearing in $\boldsymbol{V}, \boldsymbol{W}_{j}$, and $\boldsymbol{U}$ are all perpendicular to each other. Thus

$$
\begin{aligned}
\left(t_{1}, \cdots, t_{j} ;[\boldsymbol{V}, \boldsymbol{x}] ;\left[\boldsymbol{W}_{1}, \boldsymbol{y}_{1}\right], \cdots,\left[\boldsymbol{W}_{j}, \boldsymbol{y}_{j}\right] ;[\boldsymbol{U}, \boldsymbol{z}]\right) \\
\in \Delta^{j} \times B_{j}^{\perp}(k u(X ; H), k u(Y ; H), k u(Z ; H)) .
\end{aligned}
$$

It is elementary to check the defining equivalence relation of $k u\left(M_{f, g}\right)$ is compatible with the simplicial relation under the above correspondence and we obtain a homeomorphism

$$
\pi: k u\left(M_{f, g} ; H\right) \longrightarrow\left|B_{*}^{\perp}(k u(X ; H), k u(Y ; H), k u(Z ; H))\right| .
$$

Thus Theorem 7 is a corollary to the following fact.

Theorem 9. Given continuous maps

$$
X \stackrel{f}{\longleftarrow} Y \stackrel{g}{\longrightarrow} Z,
$$

we have the following quasifibration

$$
\begin{aligned}
k u(Z ; H) \longrightarrow \mid B_{*}^{\perp}(k u(X ; H), k u(Y ; H), & k u(Z ; H)) \mid \\
& \longrightarrow\left|B_{*}^{\perp}(k u(X ; H), k u(Y ; H), *)\right|
\end{aligned}
$$

We can prove this theorem by mimicing the proof of Theorem 8 . However, it will be a waste of time to write down a proof which is almost identical to that of Theorem 8 . It is natural to expect that there is a uniform way which proves both Theorem 8 and Theorem 9 at the same time. Such a proof would be useful for other homology theories including the twisted $K$-homology theory.

Notice that $k u(X ; H)$ is a "partial monoid", i.e. products are defined on certain pairs of elements. We can extend the two-sided bar construction to partial monoids.

Definition 25. A partial (topological) monoid is a pointed topological space $(M, *)$ together with a subspace $C(M) \subset M \times M$ and a map

$$
\mu_{M}: C(M) \longrightarrow M
$$

satisfying the following conditions:

1. $\left(m_{1}, m_{2}\right) \in C(M)$ and $\left(\mu_{M}\left(m_{1}, m_{2}\right), m_{3}\right) \in C(M)$ if and only if $\left(m_{2}, m_{3}\right) \in$ $C(M)$ and $\left(m_{1}, \mu_{M}\left(m_{2}, m_{3}\right)\right) \in C(M)$. And

$$
\mu_{M}\left(\mu_{M}\left(m_{1}, m_{2}\right), m_{3}\right)=\mu_{M}\left(m_{1}, \mu_{M}\left(m_{2}, m_{3}\right)\right)
$$

for such triple $\left(m_{1}, m_{2}, m_{3}\right) \in\left(\mu_{M} \times 1\right)^{-1}(C(M))=\left(1 \times \mu_{M}\right)^{-1}(C(M))$. 
2. $M \vee M \subset C(M)$ and the following diagram is commutative

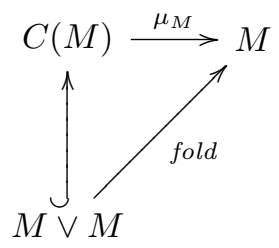

The set $C(M)$ is called the set of composable pairs.

Definition 26. Let $M$ be a partial monoid. A left action of $M$ on a space $X$ is $\operatorname{map}$

$$
\mu_{X}: C_{M}(X) \longrightarrow X
$$

where $C_{M}(X) \subset M \times X$, satisfying the following conditions:

1. $\left(m_{2}, x\right) \in C_{M}(X)$ and $\left(m_{1}, \mu_{X}\left(m_{2}, x\right)\right) \in C_{M}(X)$ if and only if $\left(m_{1}, m_{2}\right) \in$ $C(M)$ and $\left(\mu_{M}\left(m_{1}, m_{2}\right), x\right) \in C_{M}(X)$. And

$$
\mu_{X}\left(m_{1}, \mu_{X}\left(m_{2}, x\right)\right)=\mu_{X}\left(\mu_{M}\left(m_{1}, m_{2}\right), x\right) .
$$

2. $\{*\} \times X \subset C_{M}(X)$ and the following diagram is commutative

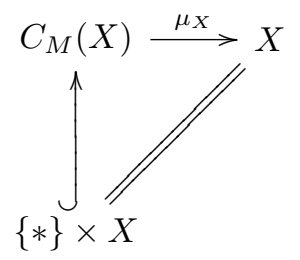

$C_{M}(X)$ is also called the set of composable pairs.

$A$ right action is defined similarly.

Definition 27. For a partial monoid $M$ acting on $X$ and $Y$ from the right and the left, respectively, define a subspace

$$
B_{n}(X, M, Y) \subset X \times M^{n} \times Y
$$

as the set of $\left(x, m_{1}, \cdots, m_{n}, y\right)$ satisfying

$$
\left(x, m_{1}\right) \in C_{M}(X),\left(m_{1}, m_{2}\right) \in C(M), \cdots,\left(m_{n}, y\right) \in C_{M}(Y) .
$$

The collection $B_{*}(X, M, Y)=\left\{B_{n}(X, M, Y)\right\}_{n \geq 0}$ forms a simplicial space. The following Theorem proves Theorem 9 .

Theorem 10. Let $M$ be a partial topological monoid with a good unit acting on $X$ and $Y$ from the right and the left, respectively. If the inclusions 


$$
\begin{aligned}
B_{n}(X, M, Y) & \hookrightarrow X \times M^{n} \times Y \\
B_{n}(X, M, *) & \hookrightarrow X \times M^{n} \\
C_{M}(Y) & \hookrightarrow M \times Y
\end{aligned}
$$

are weak equivalences for each $n$ and if the action of $m \in M$ on $Y$ induces a weak equivalence

$$
Y_{m}=\left\{y \in Y \mid(m, y) \in C_{M}(Y)\right\} \stackrel{m \cdot}{\longrightarrow} Y
$$

for each $m \in M$, then

$$
p^{Y}:\left|B_{*}(X, M, Y)\right| \longrightarrow\left|B_{*}(X, M, *)\right|
$$

is a quasifibration.

Proof. See Tam].

Theorem 9 is now a corollary to this theorem and the followng elementary but important property of $\stackrel{\perp}{\times}$ on $k u(X ; H)$.

Lemma 7. The inclusion

$$
j: k u(X ; H) \stackrel{\perp}{\times} k u(Y ; H) \hookrightarrow k u(X ; H) \times k u(Y ; H)
$$

induces a natural homotopy equivalence.

Proof. Choose an isometry

$$
\varphi: H \oplus H \longrightarrow H
$$

and consider the following composition

$$
\begin{aligned}
& \widetilde{\varphi}: k u(X ; H) \times k u(Y ; H)=k u(X ; H \oplus 0) \times k u(Y ; 0 \oplus H) \\
& \stackrel{i_{1 *} \times i_{2} *}{\longrightarrow} k u(X ; H \oplus H) \stackrel{\perp}{\times} k u(Y ; H \oplus H) \\
& \stackrel{\varphi_{*} \times \varphi_{*}}{\longrightarrow} k u(X ; H) \stackrel{\perp}{\times} k u(Y ; H) .
\end{aligned}
$$

Let us show this $\widetilde{\varphi}$ is a homotopy inverse to the inclusion $j$.

$j \circ \widetilde{\varphi} \simeq 1$ is easy. We can use a linear isotopy between the identity on $H$ and the compositions

$$
\begin{aligned}
& H=H \oplus 0 \hookrightarrow H \oplus H \stackrel{\varphi}{\longrightarrow} H \\
& H=0 \oplus H \hookrightarrow H \oplus H \stackrel{\varphi}{\longrightarrow} H
\end{aligned}
$$

on each component.

In order to prove $\tilde{\varphi} \circ j \simeq 1$, note that there is an isotopy $F$ from $i_{1}$ to $i_{2}$ in $H \oplus H$ which satisfies the following condition: 


$$
V \perp W \Longrightarrow F(V, t) \perp i_{2}(W) \text { for all } t \in I .
$$

Thus we have homotopies in $k u(X ; H) \stackrel{\perp}{\times} k u(Y ; H)$

$$
\widetilde{\varphi} \circ j=\varphi_{*} \circ i_{1 *} \times \varphi_{*} \circ i_{2 *} \simeq \varphi_{*} \circ i_{2 *} \times \varphi_{*} \circ i_{2 *} \simeq 1 .
$$

Let us consider the twisted version

$$
k u^{A S}(-; H): \operatorname{Spaces}_{B P U(H)} \longrightarrow \text { Spaces }_{*} .
$$

We need a fiberwise version of $\stackrel{\perp}{\times}$. Recall that we need to work in the category of $\mathcal{L}(1 ; H)$-spaces to define $k u^{A S}(-; H)$. Let us first fix an expression of elements of $k u^{A S}(\varphi, s ; H)$.

Definition 28. For an object $(\varphi, s)$ in $\operatorname{Spaces}_{B P U(H)}$ with $\varphi: X \rightarrow B P U(H)$, write an element of $k u^{A S}(\varphi, s ; H)$ as $\left[f ; e_{1}, \cdots, e_{j}\right]$, where $f \in \mathcal{L}(j)$ and

$$
e_{i} \in E_{\varphi}(\mathbb{P}(H))=\varphi^{*} E P U(H) \times_{P U(H)} \mathbb{P}(H) .
$$

By the base point relation we may assume that none of $e_{i}$ 's belong to $s(B P U(H))$. $B y$ the Grassmannian relation, we may arrange $e_{1}, \cdots, e_{j}$ so that

$$
\left(p_{\varphi}\left(e_{1}\right), \cdots, p_{\varphi}\left(e_{j}\right)\right)=\left(\Delta^{k_{1}}\left(x_{1}\right), \cdots, \Delta^{k_{m}}\left(x_{m}\right)\right)
$$

for $x_{i} \in X$ and $x_{i} \neq x_{i^{\prime}}$ if $i \neq i^{\prime}$. Thus there exist a partition of $\{1, \cdots, j\}$

$$
S_{1} \amalg \cdots \amalg S_{m}=\{1, \cdots, j\}
$$

such that

$$
e_{i}=\left[x_{k}, u_{i}\right]
$$

for some $u_{i} \in \operatorname{EP}(H)$ if $i \in S_{k}$, where

$$
E \mathbb{P}(H) \longrightarrow B P U(H)
$$

is the universal $\mathbb{P}(H)$-bundle over $B P U(H)$.

With these representatives, we denote $\left[f ; e_{1}, \cdots, e_{j}\right]$ by

$$
\left[f ; x_{1}, \cdots, x_{m} ; u_{1}, \cdots, u_{j}\right]
$$

or $[f ; \boldsymbol{x} ; \boldsymbol{u}]$. We call such an expression a normalized form.

Definition 29. Define a subspace

$$
k u^{A S}(\varphi, s ; H) \stackrel{\perp}{\times} k u^{A S}(\psi, t ; H) \subset k u^{A S}(\varphi, s ; H) \times k u^{A S}(\psi, t ; H)
$$

as follows: for $[f ; \boldsymbol{x} ; \boldsymbol{u}] \in k u^{A S}(\varphi, s ; H)$ and $[g ; \boldsymbol{y} ; \boldsymbol{v}] \in k u^{A S}(\psi, t ; H)$ in normalized forms with associated partitions $S$ and $T$, define $([f ; \boldsymbol{x} ; \boldsymbol{u}],[g ; \boldsymbol{y} ; \boldsymbol{v}]) \in$ $k u^{A S}(\varphi, s ; H) \stackrel{\perp}{\times} k u^{A S}(\psi, t ; H)$ if and only if

$$
f\left(\left\langle u_{i} \mid i \in S_{k}\right\rangle\right)=g\left(\left\langle v_{i^{\prime}} \mid T_{k^{\prime}}\right\rangle\right)
$$

for any $k$ and $k^{\prime}$ with $\varphi\left(x_{k}\right)=\psi\left(y_{k^{\prime}}\right)$. 
The operation $\stackrel{\perp}{\times}$ is associative and we may form the following analogue of the bar construction.

Definition 30. Given morphisms

$$
(\varphi, s) \stackrel{f}{\longleftarrow}(\psi, t) \stackrel{g}{\longrightarrow}(\zeta, u)
$$

in Spaces $_{B P U(H)}$, define

$$
\begin{aligned}
&\left.B_{n}^{\perp}\left(k u^{A S}(\varphi, s) ; H\right), k u^{A S}(\psi, t ; H), k u^{A S}(\zeta, u ; H)\right) \\
&=k u^{A S}(\varphi, s ; ; H) \stackrel{\perp}{\times} k u^{A S}(\psi, t ; H)^{\stackrel{\perp}{\times} n} \stackrel{\perp}{\times} k u^{A S}(\zeta, u ; ; H) .
\end{aligned}
$$

We obtain a simplicial space $\left.B_{*}^{\perp}\left(k u^{A S}(\varphi, s) ; H\right), k u^{A S}(\psi, t ; H), k u^{A S}(\zeta, u ; H)\right)$.

We can take mapping cylinders and mapping cones in the category Spaces $_{B P U(H)}$ and we have the following identification. The proof is an obvious modification of that of Lemma 6 and is omitted.

Lemma 8. Given morphisms

$$
(\varphi, s) \stackrel{f}{\longleftarrow}(\psi, t) \stackrel{g}{\longrightarrow}(\zeta, u)
$$

in $\mathbf{S p a c e s}_{B P U(H)}$, we have the following natural homeomorphism

$$
\left.k u^{A S}\left(M_{f, g}\right) \cong \mid B_{*}^{\perp}\left(k u^{A S}(\varphi, s) ; H\right), k u^{A S}(\psi, t ; H), k u^{A S}(\zeta, u ; H)\right) \mid .
$$

Suppose we have maps

$$
(\varphi, s) \stackrel{f}{\longleftarrow}(\psi, t) \stackrel{g}{\longrightarrow}(\zeta, u)
$$

in $\mathbf{S p a c e s}_{B P U(H)}$. We obtain a cofibration sequence

$$
(\zeta, u) \longrightarrow M_{f, g} \longrightarrow C_{f}
$$

and a sequence

$$
\begin{aligned}
& k u^{A S}(\zeta, u ; H) \longrightarrow\left|B_{*}^{\perp}\left(k u^{A S}(\varphi, s ; H), k u^{A S}(\psi, t ; H), k u^{A S}(\zeta, u ; H)\right)\right| \\
& \longrightarrow\left|B_{*}^{\perp}\left(k u^{A S}(\varphi, s ; H), k u^{A S}(\psi, t ; H), *\right)\right| .
\end{aligned}
$$

We can make the proof of Lemma 7 fiberwise, and obtain the following result by applying Theorem 10 .

Theorem 11. Given maps

$$
(\varphi, s) \stackrel{f}{\longleftarrow}(\psi, t) \stackrel{g}{\longrightarrow}(\zeta, u),
$$

we have the following quasifibration

Corollary 5. The functor $k u^{A S}(-; H)$ is linear. 


\section{References}

AH59. M. F. Atiyah and F. Hirzebruch. Riemann-Roch theorems for differentiable manifolds. Bull. Amer. Math. Soc., 65:276-281, 1959.

AS69. M. F. Atiyah and I. M. Singer. Index theory for skew-adjoint Fredholm operators. Inst. Hautes Études Sci. Publ. Math., (37):5-26, 1969.

AS04. Michael Atiyah and Graeme Segal. Twisted K-theory. Ukr. Mat. Visn., 1(3):287-330, 2004, arXiv:math.KT/0407054.

Ati61. M. F. Atiyah. Thom complexes. Proc. London Math. Soc. (3), 11:291310, 1961.

BD82. Paul Baum and Ronald G. Douglas. $K$ homology and index theory. In Operator algebras and applications, Part I (Kingston, Ont., 1980), volume 38 of Proc. Sympos. Pure Math., pages 117-173. Amer. Math. Soc., Providence, R.I., 1982.

BD98. John C. Baez and James Dolan. Categorification. In Higher category theory (Evanston, IL, 1997), volume 230 of Contemp. Math., pages 1-36. Amer. Math. Soc., Providence, RI, 1998, arXiv:math/9802029.

BJS03. Ulrich Bunke, Michael Joachim, and Stephan Stolz. Classifying spaces and spectra representing the $K$-theory of a graded $C^{*}$-algebra. In Highdimensional manifold topology, pages 80-102. World Sci. Publ., River Edge, NJ, 2003.

Bro62. Edgar H. Brown, Jr. Cohomology theories. Ann. of Math. (2), 75:467484, 1962. Correction in Ann. of Math., vol. 78 (1963), p. 201.

Bro65. Edgar H. Brown, Jr. Abstract homotopy theory. Trans. Amer. Math. Soc., 119:79-85, 1965.

CJ98. Michael Crabb and Ioan James. Fibrewise homotopy theory. Springer Monographs in Mathematics. Springer-Verlag London Ltd., London, 1998.

DB86. Robert S. Doran and Victor A. Belfi. Characterizations of $C^{*}$-algebras, volume 101 of Monographs and Textbooks in Pure and Applied Mathematics. Marcel Dekker Inc., New York, 1986. The Gel'fand-Naŭmark theorems.

DK70. P. Donovan and M. Karoubi. Graded Brauer groups and $K$-theory with local coefficients. Inst. Hautes Études Sci. Publ. Math., (38):5-25, 1970.

Dou06. Christopher L. Douglas. On the twisted $K$-homology of simple Lie groups. Topology, 45(6):955-988, 2006, arXiv:math.AT/0402082.

DS95. W. G. Dwyer and J. Spaliński. Homotopy theories and model categories. In Handbook of algebraic topology, pages 73-126. North-Holland, Amsterdam, 1995.

DT58. Albrecht Dold and René Thom. Quasifaserungen und unendliche symmetrische Produkte. Ann. of Math. (2), 67:239-281, 1958.

EKMM97. A. D. Elmendorf, I. Kriz, M. A. Mandell, and J. P. May. Rings, modules, and algebras in stable homotopy theory, volume 47 of Mathematical Surveys and Monographs. American Mathematical Society, Providence, RI, 1997.

ES52. Samuel Eilenberg and Norman Steenrod. Foundations of algebraic topology. Princeton University Press, Princeton, New Jersey, 1952.

Goo03. Thomas G. Goodwillie. Calculus. III. Taylor series. Geom. Topol., 7:645711 (electronic), 2003. 
Hir03. Philip S. Hirschhorn. Model categories and their localizations, volume 99 of Mathematical Surveys and Monographs. American Mathematical Society, Providence, RI, 2003.

Hov99. Mark Hovey. Model categories, volume 63 of Mathematical Surveys and Monographs. American Mathematical Society, Providence, RI, 1999.

HSS00. Mark Hovey, Brooke Shipley, and Jeff Smith. Symmetric spectra. J. Amer. Math. Soc., 13(1):149-208, 2000.

Kar68. Max Karoubi. Algèbres de Clifford et K-théorie. Ann. Sci. École Norm. Sup. (4), 1:161-270, 1968.

May75. J. Peter May. Classifying spaces and fibrations. Mem. Amer. Math. Soc., 1(1, 155):xiii 98, 1975.

Mil67. R. James Milgram. The bar construction and abelian $H$-spaces. Illinois J. Math., 11:242-250, 1967.

Mit. Paul D. Mitchener. $K K$-theory spectra for $C^{*}$-categories and discrete groupoid $C^{*}$-algebras, arXiv:0711.2152.

MM02. M. A. Mandell and J. P. May. Equivariant orthogonal spectra and $S$ modules. Mem. Amer. Math. Soc., 159(755):x 108, 2002.

MS06. J. P. May and J. Sigurdsson. Parametrized homotopy theory, volume 132 of Mathematical Surveys and Monographs. American Mathematical Society, Providence, RI, 2006, arXiv:math.AT/0411656

Poi96. Henri Poincaré. Euvres. Tome VI. Les Grands Classiques GauthierVillars. [Gauthier-Villars Great Classics]. Éditions Jacques Gabay, Sceaux, 1996. Géométrie. Analysis situs (topologie). [Geometry. Analysis situs (topology)], Reprint of the 1953 edition.

Qui67. Daniel G. Quillen. Homotopical algebra. Lecture Notes in Mathematics, No. 43. Springer-Verlag, Berlin, 1967.

Ros89. Jonathan Rosenberg. Continuous-trace algebras from the bundle theoretic point of view. J. Austral. Math. Soc. Ser. A, 47(3):368-381, 1989.

RS06. Rui M.G. Reis and Richard J. Szabo. Geometric K-Homology of Flat D-Branes. Commun. Math. Phys., 266:71-122, 2006, arXiv:hepth/0507043.

RSV. Rui M.G. Reis, Richard J. Szabo, and Alessandro Valentino. KOHomology and Type I String Theory. HWM-06-40, EMPG-06-09, arXiv:hep-th/0610177.

Seg74. Graeme Segal. Categories and cohomology theories. Topology, 13:293312, 1974.

Seg77. Graeme Segal. $K$-homology theory and algebraic $K$-theory. In $K$-theory and operator algebras (Proc. Conf., Univ. Georgia, Athens, Ga., 1975), pages 113-127. Lecture Notes in Math., Vol. 575. Springer, Berlin, 1977.

Shi01. Kazuhisa Shimakawa. Configuration spaces with partially summable labels and homology theories. Math. J. Okayama Univ., 43:43-72, 2001.

Shi07. Kazuhisa Shimakawa. Labeled configuration spaces and group completions. Forum Math., 19(2):353-364, 2007.

Tam. Dai Tamaki. Two-sided bar constructions for partial monoids and applications to $K$-homology theory. this volume.

Tho54. René Thom. Quelques propriétés globales des variétés différentiables. Comment. Math. Helv., 28:17-86, 1954.

Wal. Robert Waldmüller. Products and push-forwards in parametrised cohomology theories, arXiv:math.AT/0611225. 
Wan. Bai-Ling Wang. Geometric cycles, index theory and twisted $K$ homology, arXiv:0710.1625.

Whi62. George W. Whitehead. Generalized homology theories. Trans. Amer. Math. Soc., 102:227-283, 1962.

Wit98. Edward Witten. D-branes and K-theory. J. High Energy Phys., (12):Paper 19, 41 pp. (electronic), 1998, arXiv:hep-th/9810188. 